\title{
A conserved ATG2-GABARAP interaction is critical for phagophore closure.
}

Mihaela Bozic ${ }^{1}$, Luuk van den Bekerom ${ }^{1}$, Beth A. Milne ${ }^{1}$, Nicola Goodman ${ }^{1}$, Lisa Roberston ${ }^{1}$, Alan R. Prescott ${ }^{2}$, Thomas J. Macartney ${ }^{3}$, Nina Dawe ${ }^{1}$ \& David G. McEwan ${ }^{*}$

1. Division of Cell Signalling \& Immunology, School of Life Sciences, University of Dundee, Dundee, UK.

2. Dundee Imaging Facility, School of Life Sciences, University of Dundee, Dundee, UK.

3. MRC Protein Phosphorylation and Ubiquitylation Unit, School of Life Sciences, University of Dundee, Dundee, UK

${ }^{*}$ Correspondence to: d.g.mcewan@dundee.ac.uk 


\begin{abstract}
The intracellular trafficking pathway, macroautophagy, acts as a recycling and disposal service that can be upregulated during periods of stress, to maintain cellular homeostasis. An essential transition point in the pathway is the sealing of the immature phagophore to form an autophagosome, isolating unwanted cargo prior to lysosomal degradation. However, little mechanistic detail is known about phagophore closure. Human ATG2A and ATG2B proteins, through their interaction with WIPI proteins, are thought to be key players during phagophore closure. We have identified a highly-conserved motif driving the interaction between human ATG2 and GABARAP proteins that is in close proximity to the ATG2-WIPI4 interaction site. We show that the ATG2-GABARAP interaction mutants are unable to close phagophores resulting in blocked autophagy, similar to ATG2A/ATG2B double knock-out cells. In contrast, the ATG2-WIPI4 interaction mutant fully restored phagophore closure and autophagy flux, similar to wild type ATG2. Taken together, we provide new mechanistic insights to the requirements for ATG2 function at the phagophore and suggest that an ATG2-GABARAP interaction is essential for phagophore closure, whereas ATG2-WIPI4 interaction is dispensable.
\end{abstract}

Key words: Autophagy; phagophore; autophagosome; GABARAP; ATG2

\begin{abstract}
Abbreviations: ATG2 - Autophagy-related protein 2; ESCRT - endosomal sorting complex required for transport; GABARAP - Gamma-aminobutyric acid receptor-associated protein: GIM - GABARAP Interaction Motif; GST - Glutathione S-transferase; LIR - LC3 interaction region; MAP1LC3 (LC3) - Microtubule-associated proteins 1A/1B light chain; PtIns3P phosphatidylinositol-3-phosphate; PI3K - PtsIns3P kinase; SQSTM1 - Sequestosome-1; VPS34 - Vacuolar Protein sorting 34; WIPI - WD repeat domain phosphoinositide-interacting protein;
\end{abstract}




\section{Introduction.}

How our cells ability to deal with a wide variety of cellular stresses is depends on two quality control pathways - namely the ubiquitin proteasome and the autophagosome to lysosome (macroautophagy) pathways. Both act in concert to ensure that our cells maintain their homeostasis. Macroautophagy (henceforth autophagy) is a multi-step process that requires the initiation and formation of a phagophore that grows and surrounds cargo to be degraded. The phagophore eventually seals to form a double-membraned vesicle, termed autophagosome. The autophagosome is then transported to, and fuses with, the lysosome where the inner autophagosomal membrane along with the cargo contents are degraded and recycled back to the cell (reviewed in [1]). This provides an intracellular pool of amino acids and lipids that the cell can utilize under periods of stress. Autophagy inducing stresses include amino acid/growth factor starvation (non-selective, bulk autophagy), mitochondrial depolarization[2, 3], pathogen invasion [4] and protein aggregate accumulation[5] (selective autophagy). In all cases, the inclusion of the cargo within the growing phagophore, and eventually the autophagosome, serves to isolate potentially cytotoxic material from the surrounding intracellular environment.

The molecular machinery involved in autophagosome formation is extensive and, for the most part, highly conserved. More than 30 ATG (스니ophaGy) genes regulate all stages of autophagosome formation; from initiation, cargo selection, transport to fusion with the lysosome. In higher eukaryotes, several kinase complexes, as well as ubiquitin-like conjugation machinery, are required for the initiation and expansion of the autophagosome. For example, the initiation kinase complex consists of ULK1/ATG13/ATG101/FIP200 and the lipid kinase complex VPS34/Beclin1/ATG14L1/p150 [6-8]. To grow the autophagosome and to recruit cargo requires the ubiquitin-like conjugation machinery, consisting of ATG7 (E1like) ATG3 and ATG10 (E2-like) and ATG12-ATG5-ATG16L1 (E3-like complex), which are responsible for the conjugation of ubiquitin-like MAP1LC3/GABARAPs (mammalian homologues of yeast Atg8) to phosphatidylethanolamine (PE) on the growing phagophore membrane [9]. LC3/GABARAP proteins, once conjugated to PE, can localize to both the inner and outer autophagosomal membrane. This allows the ATG8s to interact with proteins containing an LC3 interaction region/GABARAP Interaction Motif (LIR/GIM), linking the phagophore to cargo or the phagophore/autophagosome to the cellular transport and fusion machinery [10-13]. The majority of LIR motifs contain a core $\Theta-X_{1}-X_{2}-\Gamma$ motif, where $\Theta$ is an aromatic residue $(\mathrm{W} / \mathrm{F} / \mathrm{Y})$, and $\Gamma$ is a large hydrophobic residue $(\mathrm{L} / \mathrm{V} / \mathrm{I})$. In addition, acidic and/or phosphorylatable serine/threonine residues $\mathrm{N}$ - and $\mathrm{C}$-terminal of the core LIR sequence can contribute to the stabilization of LIR-ATG8 interactions [14-16]. 
Despite a surge in our understanding of the mechanisms involved in autophagy, there are still questions pertaining as to how the double-membrane phagophore closes and seals to form the autophagosome. In particular, the molecular components and how they interact are relatively unknown. For example, in yeast, Vps21 (Rab5-related GTPase) and Rab5 influence phagophore closure $[17,18]$. The mammalian ATG8 protein GATE-16 (GABARAPL2) has been shown to be involved in the later stages of autophagosome biogenesis [19] and its $\mathrm{N}$-terminal extension can promote membrane fusion events, hinting at a possible role during phagophore closure[20]. However, a recent study where LC3 and GABARAPs were knocked-out, indicated that LC3/GABARAPs were not required for phagophore closure [21]. A mutant form of ATG4B (C74A), the cysteine protease responsible for LC3 and GABARAP priming and removal from the autophagosomal membrane, prevents LC3 and GABARAP lipidation and results in an increased number of unsealed phagophore membranes [22]. In addition to core autophagy proteins, a component of the ESCRT-III endocytic machinery, CHMP2A, regulates the separation of inner and outer phagophore membranes [23].

One intriguing example of the role of ATG proteins during phagophore closure is the poorly understood ATG2 proteins, ATG2A and ATG2B. Mammalian ATG2s are >1900 amino acids in length and share approximately $40 \%$ amino acid sequence homology but are only $13 \%$ similar to the single isoform of S.cerevisiae Atg2 and $24-26 \%$ to the D.melanogaster Atg2, indicating a potential divergence of function. Indeed, the reconstitution of human ATG2A in yeast $\Delta$ atg2 cells is not sufficient to restore the autophagy defects [24]. In yeast, Atg2 constitutively interacts with Atg18 at phosphatidylinositol-3-phosphate (PtIns3P)-rich membrane regions and tethers pre-autophagosomal membranes to the endoplasmic reticulum for autophagosome formation [25, 26]. Mammalian homologues of yeast Atg18 are the WIPI proteins (WIPI1-4) that are involved in various stages of autophagosome formation [27-29]. ATG2A and ATG2B preferentially interact with WIPI4 (WDR45) through a conserved Y/HFS motif [29-31]. Simultaneous depletion of both ATG2A and ATG2B results in the accumulation of small, open immature phagophore structures [32, 33]. The depletion of WIPI4 also causes open phagophore structures but they are morphologically dissimilar to those generated after ATG2A/B depletion [29]. Interestingly, previous studies have not, despite mapping the ATG2-WIPI4 interaction, shown whether this interaction is required for the restoration of autophagy flux in ATG2A/B depleted cells [29-31]. Herein, CRISPR/Cas9 was used to generate GFP-ATG2A knock-in cells as a tool to address the endogenous localization and interaction of human ATG2A. We have identified a direct interaction between the GABARAP family of mammalian ATG8 proteins and ATG2A and ATG2B that is mediated through a highly conserved LIR/GIM sequence. Surprisingly, the newly identified LIR/GIM sequence in ATG2A and ATG2B is approximately 30 amino acids $\mathrm{N}$-terminal of the 
WIPI4 interaction motif and represent independent interaction sites in the C-terminus of human ATG2s. Using reconstituted ATG2A/2B double knockout cells, we show that the disruption of ATG2-WIPI4 interaction had no discernible effects on phagophore closure and autophagy flux, whereas mutation of the LC3/GABARAP interaction motif on ATG2 completely blocked phagophore closure and autophagy flux. Taken together, these data provide new insights into essential ATG2 interactions during autophagosome biogenesis.

\section{Results and Discussion.}

\section{Endogenous GFP-tagged ATG2A co-localizes and co-precipitates with GABARAP.}

In order to study the function of endogenous ATG2 proteins, we generated GFP-tagged ATG2A knock-in U2OS cells using CRISPR/Cas9 (Figure EV1A-1C). Under complete, nutrient-rich conditions (CM), GFP-ATG2A showed a dispersed localization, with little overlap with LC3B (Figure 1A, Upper panels). However, upon starvation we observed ring and punctate structures form that localized in close proximity to LC3B positive vesicles (Figure 1A, Lower panels). Endogenous ATG2B co-localized with GFP-ATG2A on both the punctate and ring-like structures observed (Figure 1A, lower panels). Endogenous GFPATG2A co-localized with early autophagy marker proteins WIPI2 (Figure 1B) and ATG16L1 (Figure 1C) at LC3B positive structures formed under starvation conditions. In addition, GABARAP-L1 was also present on GFP-ATG2A/LC3B positive structures under starvation conditions (Figure 1D). Given the presence of both GABARAP-L1 and LC3B co-localizing with GFP-ATG2A, we were curious as to whether we could co-precipitate either or both using GFP-ATG2A as bait. Using U2OS WT (control) or GFP-ATG2A U2OS cell under CM or starvation conditions, we immunoprecipitated GFP-ATG2A. WIPI4, a cognate ATG2 interaction partner [29-31], co-precipitated with GFP-ATG2A under both CM and starvation conditions (Figure 1E). We could not detect endogenous LC3B in GFP-ATG2A immunoprecipitates; but we detected increased co-precipitation of GABARAP, under starvation conditions (Figure 1E). Indeed, both ATG2A (Figure EV1D) and ATG2B (Figure EV1E) were able to co-precipitate with GFP-tagged GABARAP but not with GFP-LC3B when overexpressed in HEK293T cells. Taken together, this indicates that endogenously tagged ATG2A is active in the autophagy pathway and both ATG2A and ATG2B may functionally interact with the GABARAP family of mammalian ATG8 proteins.

\section{Identification of a conserved LC3 interaction region sequence in ATG2A and ATG2B.}

Direct interaction with Atg8/LC3/GABARAP proteins is mediated through the presence of a LIR (LCC3 Interaction Region) on the target protein [5, 13, 34-37]. ATG2 proteins have previously been shown to be part of the mammalian LC3/GABARAP interactome [38] but no direct link interaction, or consequences, has been shown. We therefore performed an in- 
silico analysis of both ATG2A and ATG2B proteins using the iLIR tool [39], as well as manual annotation, to identify potential LIRs that conform to the $[E / D / S / T]-W / F / Y-X_{1}-X_{2}-L / I / V$ consensus sequence. We excluded potential LIR sequences present on secondary structures or within domains, as LIR sequences are most frequently found within disordered regions between domains [40]. We found that ATG2A contained five, and ATG2B contained six, potential LIRs (Table 1 and Figure EV2A). We then mutated the core sequence of all the potential LIR sequences in both ATG2A and ATG2B to alanine residues (Table 1) and tested the interaction using purified GST-tagged ATG8 proteins [5]. Out of the five potential LIRs present within ATG2A (Figure EV2B-E and Figure 2A upper) and six potential LIRs of ATG2B (Figure EV2F-G and Figure 2A lower), only a single, highly conserved functional LIR was present in both ATG2A (LIR\#5) and ATG2B (LIR\#6). Mutation of ATG2A-LIR\#5 (amino acids 1362-1365; SDEFCIL; Figure 2A, mLIR) and ATG2B-LIR\#6 (amino acids 1491-1494; NDDFCIL; Figure 2A, mLIR) resulted in the loss of GST-ATG8 interaction, compared to WT proteins (Figure 2A). Next, we overexpressed ATG2A/ATG2B-WT or LIR mutants with GFP alone, GFP-LC3B or GFP-GABARAP and immunoprecipitated the GFPtag. ATG2A-WT (Figure 2B) and ATG2B-WT (Figure 2C) co-precipitated mainly with GABARAP and this interaction was abolished when their core LIR sequence was mutated to alanine (mLIR; FCIL/AAAA; Figure 2B-2C). ATG2A had a second potential LIR sequence (aa926-929 FSTL/AAAA; mLIR\#2). However, immunoprecipitation of GFP-tagged LC3B and GABARAP showed that Myc-ATG2A-mLIR\#2 was still able to interact with GABARAP to the same extent as ATG2A-WT; whereas ATG2A-mLIR\#5 abolished the interaction (Figure EV2H).

Alignment of the amino acid sequences of ATG2 proteins from multiple species revealed that the ATG2A/2B LIR sequence is highly conserved in multiple vertebrates and invertebrates (Figure 2D, orange box). This includes organisms with a single ATG2 isoform; Drosophila melanogaster (DmAtg2), Caenorhabditis elegans (CeAtg2), and species with two ATG2 isoforms; Danio rerio (DrAtg2a/DrAtg2a) and Xenopus tropicalis (XtAtg2a/XtAtg2b). However, the LIR does not appear to be present in Saccharomyces cerevisiae or Saccharomyces pombe, indicating a potential divergence in Atg2 function (Figure 2D, orange box). Taken together, both ATG2A and ATG2B contain a single, highly conserved LIR motif that preferentially interacts with the GABARAP family of mammalian ATG8 proteins.

Since the first LC3 interaction region was identified in the prototypical autophagy receptor protein, p62/SQSTM1 [10], the number of functional LIR-containing proteins identified to date has grown considerably. The interaction between mammalian ATG8s and LIR containing proteins serves to control all aspects of the autophagy pathway, from cargo 
selection to formation, transport and fusion of the autophagosome. Not only are these interactions sequences present in mammalian, plant, fungi and invertebrate species, they are also present in a number of viral [41] and bacterial [42] proteins, potentially to aid pathogen survival and subversion of the pathway. We have identified a highly conserved LIR within both ATG2A and ATG2B that differ only in a few amino acids both N-and C-terminal of the core LIR sequence (FCIL; Figure 2D). This raises an ongoing question as to how specificity within the system is achieved, particularly in mammalian systems that are complicated by the expression of six LC3/GABARAP isoforms. We, and others [13, 43, 44], have attempted to decipher the code that dictates whether a protein with a particular LIR sequence will preferentially interact with LC3 over GABARAP. Interestingly, ATG2A and ATG2B do not conform to the recently identified GABARAP interaction motif consensus sequence (W/F-I/V-X-I/V) [13] despite preferring GABARAP over LC3 in coimmunoprecipitation from cells (Figure 1E; Figure 2B-C). Surprisingly, both ATG2A and ATG2B can also interact with LC3A (Figure 2A), however the functional consequences and the in vivo preference appears directed towards GABARAP proteins. The high degree of conservation of the ATG2A/B LIR sequence throughout vertebrates and invertebrates (Figure 2D) potentially indicates a conserved function, even in species with only a single ATG2 isoform, such as D.melanogaster and C.elegans. Therefore, understanding the role of ATG2-LC3/GABARAP interaction during autophagy will provide insights into ATG2s mechanism of function in multiple species.

\section{ATG2-WIPI interaction is not affected by mutation of the ATG2 LIR.}

Intriguingly, the recently described WIPI4 interaction region that contains an essential Y/HFS motif $[30,31]$ is approximately 30 amino acids C-terminal of the newly identified LIR sequence in both ATG2A and ATG2B (Figure 2D, purple box). Therefore, we wanted to test whether the interaction between ATG2, GABARAP and WIPI4 were co-dependent, or whether they represented independent interactions. The YFS motif found in ATG2A (amino acids 1395-1397; YFS/AAA; mYFS) was mutated and HA-tagged ATG2A-WT, -mLIR or $m Y F S$ variants were immunoprecipitated from cell lysates and probed for the presence of GFP-tagged GABARAP and endogenous WIPI4. ATG2A-WT co-precipitated with both WIPI4 and GFP-GABARAP under complete media (CM) and starvation conditions (Figure 2E). ATG2A LIR mutant failed to co-precipitate GFP-GABARAP, whereas the WIPI4 interaction was unaffected (Figure 2E; mLIR). Conversely, mutation of the ATG2A YFS motif resulted in the loss of WIPI4 interaction but not GABARAP (Figure 2E; mYFS). Interestingly, the loss of the WIPI4 interaction site on ATG2A resulted in increased coprecipitation of lipidated GABARAP with ATG2A-mYFS (Figure 2E). Thus, within a 30 amino acid stretch mammalian ATG2 proteins are two distinct and independent interaction motifs 
that can potentially regulate ATG2 function at the growing phagophore membrane. It remains to be seen whether either or both, interactions are necessary for ATG2 function during autophagy.

\section{ATG2 LIR mutant but not WIPI4 mutant is sufficient to block autophagy.}

ATG2A can simultaneously interact with both GABARAP and WIPI4 (Figure 2E). Interestingly, previous reports that identified the WIPI4 interaction region on ATG2 [30, 31] and the ATG2 interaction site of WIPI4 [29] did not address the role of these interaction mutants during autophagy flux. Considering the close proximity of both GABARAP and WIPI4 motifs on ATG2, we wanted to dissect the individual roles of ATG2-GABARAP and ATG2-WIPI4 interactions during autophagy. Firstly, ATG2A/ATG2B double-knockout (DKO) U2OS cells were generated, that could be reconstituted with WT and mutant genes. In ATG2A/2B DKO cells, endogenous ATG2A and ATG2B were depleted compared to WT and p62/SQSTM1, lipidated mammalian ATG8s (LC3A, LC3B, LC3C, GABARAP-L1 and GABARAP-L2) were markedly increased compared to WT U2OS cells (Figure EV2I). Next, stable expression of the tandem tagged-LC3B autophagy reporter (mCherry-GFP-LC3B; [45]) in ATG2A/B DKO cells was used to assess LC3B transition from autophagosomes $(G F P+v e / m C h e r r y+v e)$ to autolysosomes (GFP-ve/mCherry+ve) due to GFP quenching at low $\mathrm{pH}$ [45]. Using confocal imaging and flow cytometry to quantify, tandem-tagged LC3B puncta in ATG2A/2B DKO cells under complete medium (CM) or starvation conditions, were both GFP and mCherry positive (Figure $\mathbf{3 A}$ and quantified in $3 \mathbf{B}$ ). This indicated that the ATG2A/B DKO U2OS cells had impaired autophagy flux, consistent with previous work [46]. Stable reconstitution of tandem-LC3B expressing ATG2A/B DKO cells with ATG2A-WT resulted in more mCherry only positive cells/puncta in complete medium (CM) conditions that were increased upon starvation (Figure 3A and quantified in 3B). However, this effect was nullified using BafliomycinA1 (to prevent lysosome acidification and quenching of GFP signal) (Figure 3A and quantified in 3B). Surprisingly, expression of ATG2A-mLIR resulted in a complete lack $m$ Cherry only puncta/cells, indicative of impaired autolysosome formation and closely resembled the $A T G 2 A / B$ DKO cells (Figure $3 A$ and quantified in $3 \mathbf{B}$ ). Unexpectedly, the ATG2A-mYFS (WIPI4 mutant) was able to fully restore autophagy flux, as indicated by increased mCherry positive puncta/cells and was comparable to the ATG2AWT results (Figure $3 \mathrm{~A}$ and quantified in $3 \mathrm{~B}$ ).

Next, we analysed the effect of ATG2A-WT, -mLIR and -mYFS expression on both p62/SQSTM1 and LC3B protein levels, as these are autophagy substrates and are good indicators of flux [47]. Stable expression of HA-tagged ATG2A-WT in ATG2A/2B DKO cells resulted in decreased p62/SQSTM1 and LC3B-II levels, compared to DKO alone, indicating rescue of the pathway and restoration of autophagy flux (Figure 3C-E, WT lane). Consistent 
with the tandem-tagged LC3B reporter assay (Figure 3A-B), expression of ATG2A-mYFS was able to fully restore autophagy flux under nutrient-rich (CM, complete medium), starvation and bafilomycin treatment (Figure 3C-E). Excitingly, the expression of ATG2AmLIR failed to rescue the defect in p62/SQSTM1 and LC3B-II (Figure 3C-E). In DKO plus ATG2A-WT and DKO plus ATG2A-mYFS expressing cells, LC3B was present within LAMP2 positive vesicles (lysosomes) after starvation plus BafliomycinA1 treatment, to induce autophagosome formation but halt their degradation (Figure EV3A, open arrows). In stark contrast, LC3B was observed juxtaposed to LAMP2 vesicles in both ATG2A/2B DKO and DKO plus ATG2A-mLIR (Figure EV3A, closed arrows), indicating an autophagosome maturation defect and consistent with the tandem-LC3B reporter assay (Figure 3A-B). One aspect of mammalian ATG2 function is the regulation of the size and distribution of lipid droplets (LDs) [33]. ATG2A localizes to the limiting membrane of LDs [33, 46]. Importantly, both HA-tagged ATG2A-mLIR and ATG2A-mYFS, as well as HA-ATG2A-WT, are able to localize to lipid droplets induced by oleate, a fatty acid supplement that induces the accumulation of neutral lipids into LDs (Figure EV3B-C). Therefore, disruption of either the ATG2A-GABARAP or ATG2A-WIPI4 interaction does not affect ATG2A localization to LDs. Taken together, our data shows that mutation of a conserved LC3/GABARAP interaction motif on ATG2A fails to restore the autophagy defect of ATG2A/ATG2B double knock out cells; whereas, the interaction with WIPI4 is dispensable for autophagy flux.

\section{ATG2-GABARAP interaction is essential for phagophore closure.}

Previous work has shown that early autophagy marker proteins accumulate on LC3B positive structures in ATG2A/B depleted cells [33]. Due to the similarities between the ATG2A/B DKO and ATG2A-mLIR phenotypes, we stained for the presence of several autophagy marker proteins under starvation conditions. ATG2A/2B DKO and DKO cells expressing ATG2A-mLIR exhibited large LC3B-positive/p62-positive structures (Figure EV4A, closed arrows) and accumulated ATG9A (Figure EV4B, closed arrows), WIPI2 (Figure EV4C, closed arrows) and ATG16L1 (Figure EV4D, closed arrows). In contrast, ATG2A/B DKO cells reconstituted with ATG2A-WT or ATG2A-mYFS resulted in vesicular LC3 and punctate p62/SQSTM1 structures (Figure EV4A, open arrows), juxtanuclear ATG9A localization (Figure EV4B, open arrows) and punctate WIPI2 (Figure EV4C, open arrows) and ATG16L1 (Figure EV4D, open arrows) consistent with restoration of efficient autophagosome biogenesis and autophagy flux.

In cells expressing ATG2A-mLIR, LC3B is lipidated (LC3B-ii; Figure 3C), early phagophore associated proteins are present (ATG9A and ATG16L1, Figure EV4B and D respectively) and the membranes contain PI3P (inferred by the presence of WIPI2, Figure EV4C). This 
indicates that the observed structures (Figure EV4 A-D) are phagophores or autophagosomes.

The mammalian ATG2 proteins have been suggested to function at the transition stage of phagophore to autophagosome maturation, the closure step [32, 33]. Therefore, in order to address the functional significance of the ATG2 interaction with GABARAP, used two assays to distinguish between phagophores and autophagosomes - a proteinase $\mathrm{K}$ protection assay (Figure 4A(i)) and Syntaxin17 (STX17) translocation (Figure 4A(ii)). Firstly, using the proteinase $\mathrm{K}$ limited proteolysis assay, which degrades proteins not protected within a membrane compartment (Figure 4A (i)), we tested whether the expression of ATG2A LIR mutant resulted in defective phagophore closure. ATG2A/B DKO cells and DKO cells reconstituted with ATG2A-WT, -mLIR or -mYFS (Figure 4B) were left in CM or starved for four hours in the presence of BafilomycinA1 (Starve+BafA1) to accumulate autophagosomes. Cells were then permeabilised using digitonin and incubated in buffer only, proteinase $\mathrm{K}$ or proteinase $\mathrm{K}$ plus Triton X-100 (to permeabilise membranes). Under $\mathrm{CM}$ conditions, the majority of the autophagy substrate p62 was degraded in all samples (Figure 4B, upper panel). After starvation plus BafilomycinA1 treatment, DKO cells reconstituted with ATG2A-WT and ATG2A-mYFS showed a large proportion of p62 resistant to proteinase $\mathrm{K}$ degradation (Figure 4B, lower panel; $38 \%$ and $56 \%$ respectively). However, p62 in both DKO and DKO plus ATG2A-mLIR cells was sensitive to proteinase $\mathrm{K}$ digestion (Figure 4B, lower panel; $18 \%$ and $17 \%$ respectively), which is indicative of immature/unsealed autophagosomes.

Next, STX17 translocation to LC3B positive vesicles in the ATG2A-WT and mutantexpressing cells was tested. STX17 translocates from the ER to fully formed autophagosomes, but not phagophores, prior to their fusion with the lysosome (Figure 4A (ii))[48]. Stable expression of GFP-syntaxin17 in the reconstituted ATG2A/B DKO cells revealed that STX17 can efficiently localize to, and surround, LC3B positive structures in both ATG2A-WT and ATG2A-mYFS expressing cells after starvation plus bafilomycinA1 treatment (Figure 4C, open arrows and quantified in Figure 4D). Conversely, in ATG2A/B DKO and DKOs plus ATG2A-mLIR cells, GFP-STX17 localized mainly to ER and punctate structures with few GFP-STX17+ve/LC3B+ve vesicles observed (Figure 4C, closed arrows and quantified in Figure 4D). Taken together, our data suggest that a conserved GABARAP interaction motif in both mammalian ATG2A and ATG2B proteins is essential for phagophore to autophagosome transition, and surprisingly, that the WIPI4 interaction is dispensable for this function during starvation-induced autophagy.

The formation of the autophagosome and its subsequent trafficking and fusion with the lysosome is a tightly controlled pathway with a number of essential components that allows it 
to progress in an orderly fashion. This enables the cell to liberate amino acid and lipid stores during periods of stress, target and remove intracellular pathogens or remove cytotoxic protein aggregates from the cell. Critical to this process is the ability of the cell to form a double-membraned phagophore that grows, surrounds and isolates the material to be removed. Despite recent advances in our knowledge, the mechanisms involved in phagophore closure are poorly understood. Recent work has shown that the ESCRT-III component CHMP2A regulates the separation of inner and outer phagophore membranes [23]. In addition to the ESCRT-III machinery, TRAPPC11, a member of TRAPP complexes involved in membrane trafficking, has been shown to recruit ATG2B-WIPI4 to phagophores in an ATG9A dependent manner [49]. The depletion of TRAPC11 results in a phenotype similar to that of ATG2A/B DKO and ATG2A-mLIR [49]. The mammalian ATG2 proteins, ATG2A and ATG2B, have been shown to be essential for phagophore formation and closure $[32,33,46]$ and depletion of WIPI4, a constitutive interaction partner of mammalian ATG2s, also negatively impacts on phagophore closure [29]. Herein, we have described a hitherto unidentified LC3/GABARAP interaction region on both ATG2A and ATG2B that is essential for phagophore formation and closure.

These results shed new light on the role of ATG2 during autophagosome biogenesis, and in particular, the interactions that are necessary for this process. Perhaps most surprisingly was the effect, or rather lack thereof, that the ATG2A-WIPI4 interaction mutant had on phagophore closure and autophagy flux. From yeast to fruit flies to humans, the ATG2ATG18 (WIPI) interaction is highly conserved. In yeast, Atg2-Atg18 interaction occurs independently of Atg18 binding PI3P [26], much like the ATG2A/B interaction with WIPI4 [29-31]. Recently, yeast Atg2 has been shown to contain both $\mathrm{N}$ - and C-terminal membrane binding domains that help tether Atg2 to membrane contact sites [25]. Human ATG2A has several domains that determine its ability to localize to membranes. Firstly, ATG2 has an Nterminal membrane binding region that is essential for autophagosome formation [46] that has now been shown to be a lipid transport domain [50,51]. This N-terminal lipid transport domain is thought to be essential for the transport of PE and phosphatidylserine from the ER/omegasome to the growing phagophore [50, 51]. This domain is homologous to the Vps13 lipid transport domain involved in organelle-organelle contact sites [52]. The second lipid interaction region of ATG2A is an amphipathic helix (AH; aa1750-1767), which is essential for ATG2 localization to both lipid droplets and isolation membranes and is essential for autophagy flux [46]. In addition, ATG2 has a c-terminal region (aa1830-1938 HsATG2A) that is involved in localization to lipid droplets but is dispensable for autophagy [46]. This raises an interesting question as to the role of the ATG2-WIPI4 interaction, as this was previously thought to be involved in ATG2 autophagy function. However, we have 
shown that ATG2-WIPI4 is dispensable for autophagosome formation and autophagy flux. Given that the ATG2A LIR mutant we identified has impaired autophagy flux (Figure 3) but can still localize to lipid droplets (Figure EV3B-C), we suggest that both the ATG2-AH and the ATG2-LIR are essential and the combination of both the LIR and AH helps to define the target membrane, allowing tethering and lipid transfer to facilitate efficient phagophore formation and autophagosome maturation (Figure 4E).

\section{Materials \& Methods.}

Antibodies. The antibodies used in this study as re as follows: Anti-GFP (Santa Cruz clone B-2, sc9996), anti-FlagM2 (SIGMA, F3165), anti-p62 (MBL, M162-3), anti-LC3B (clone 5F10 Nanotools, 0231-100/LC3-5F10) and anti-GABARAP (Abcam, ab109364) anti-ATG16L1 (MBL, PM040), anti-ATG9A (abcam, ab108338), anti-WIPI2 and anti-WIPI4 were kind gifts from Prof. Sharon Tooze, LC3A clone D50G8 (CST, \#4599), anti-LC3C (D1R8V; CST \# 14723), anti-GABARAP (Abcam, ab109364), anti-GATE-16 (MBL, PM038), anti-GABARAPL1 (IF, Proteintech, 11010-1-AP), anti-GABARAP-L1 (WB, Abcam, ab86497), anti-vinculin (Sigma, V9131-100UL), anti-ATG2A (Proteinech, 23226-1-AP) and ATG2B (Proteintech, 25155-1-AP), LAMP-2 (DSHB, clone H4B4), c-Myc (DSHB, clone 9E10).

Cell culture and reagents. HEK293T and U2OS or U2OS ATG2A/B double knock-out cells were maintained in Dulbecco's modified Eagle's medium (DMEM; Invitrogen 10313021) supplemented with $10 \%$ foetal bovine serum (FBS), $5 \mathrm{U} / \mathrm{ml}$ penicillin and $50 \mu \mathrm{g} / \mathrm{ml}$ streptomycin, $1 \mathrm{mM} \mathrm{L-glutamine}$ and $1 \%$ sodium pyruvate. For starvation in nutrient $\square$ deplete medium, the cells were incubated $2 \mathrm{~h}$ in Earle's balanced salt solution (EBSS; Gibco, 24010 $\square 043$ ). Bafilomycin A1 (BafA1; Enzo, BML $\square$ CM110 $\square 0100$ ) was used at $200 \mathrm{nM}$. ATG2A/2B DKO cells were stably transfected by retroviral transduction of pMSCV-Flag-HA (iTAP) vectors or lentiviral transduction of pHAGE-NTAP-mCherry or pHAGE-N-EGFP vectors. Briefly, HEK293T cells were transfected with ITAP vectors with pCG-GagPol and pCG-VSVG (retrovirus) or pHAGE constructs in combination with $\mathrm{pCMV} \square \mathrm{VSV} \square \mathrm{G}$ and psPAX2 (lentivirus) packaging vectors. Virus containing media was harvested $48 \mathrm{~h}$ post $\square$ transfection, centrifuged at $300 \mathrm{xg}$, passed through a $0.45 \square \mu \mathrm{m}$ filter and added to ATG2A/B DKO cells in the presence of $10 \mu \mathrm{g} / \mathrm{ml}$ polybrene (Sigma, H9268-5G). Transduced cells were selected by addition of $1 \mu \mathrm{g} / \mathrm{ml}$ Puromycin (iTAP and pHAGE-nTAP-mCherry) or $10 \mu \mathrm{g} / \mathrm{ml}$ Blasticidin (pHAGE-EGFP) $48 \mathrm{~h}$ after addition of viral media.

CRISPR/Cas-9 gene editing. CRISPR/Cas9-mediated deletion of ATG2A (NM_015104.3) and ATG2B (NM_018036.7) in osteosarcoma cells (U2OS) was performed by using the Cas9 D10A 'nickase' mutant and paired gRNAs approach [53] was used to target exon 1 of both ATG2A (5'- CCATGGTCAAACTGTGTGAAAGA-3' and 5'- 
TACTTGCTGCACCACTACTTAGG-3') and ATG2B (5'- CCGTTTTCGGAGTCCATCAAGAA$3^{\prime}$ and 5'-CCTGCCGGTACCTCCTGCAGAGG-3'). ATG2A and ATG2B-targeting gRNAs were transfected into $1 \times 10^{6}$ U2OS cells followed by selection with $1 \mu \mathrm{g} / \mathrm{ml}$ puromycin for $48 \mathrm{hrs}$, re-transfection, recovery (in puromycin free media) and single cell sorting to isolate clone candidates with the gene deletion.

Endogenous GFP-tagged ATG2A knock-ins were generated using a modified 'nickase' strategy (as above). Optimal sgRNA pairs were identified and chosen on the basis of being as close as possible to the point of GFP insertion while having a low combined off-targeting score (ATG2A-sgRNA1: 5'-GTCAAACTGTGTGAAAGAGC-3' \& sgRNA2: 5'AGATGTCACGATGGCTGTGGC-3'). Complementary oligos with Bbsl compatible overhangs were designed for each and these dsDNA guide inserts ligated into Bbsl-digested target vectors; the antisense guide (sgRNA2) was cloned onto the spCas9 D10A expressing pX335 vector (Addgene plasmid no. 42335) and the sense guides (sgRNA1) into the puromycin-selectable pBABED P U6 plasmid (Dundee-modified version of pBABE-puro plasmid). A donor construct consisting of GFP flanked by approximately 500 bp homology arms were synthesized by GeneArt (Life Technologies); each donor was engineered to contain sufficient silent mutations to prevent recognition and cleavage by Cas 9 nuclease. Both sgRNA and donor constructs were transfected into U2OS cells, selected in $1 \mu \mathrm{g} / \mathrm{ml}$ puromycin for 48hrs, re-transfected and allowed to recover in puromycin free complete media. When confluent, cells were single cell sorted for GFP-positive populations and homozygous clones selected for further analysis.

Western blot and immunoprecipitation. Cells (HEK293T, U2OS) were lysed in NP-40 lysis buffer (50mM TRIS, pH7.5, $120 \mathrm{mM} \mathrm{NaCl}, 1 \% \mathrm{NP}-40)$ supplemented with Complete® protease inhibitor (Roche) and phosphatase inhibitor cocktail (Roche). Lysates were passed through a 27G needle, centrifuged at 21000xg and incubated with either anti-GFP agarose (Chromotek, gta-20) or anti-HA agarose (Sigma A2095) washed 3 times in lysis buffer and subjected to SDS-PAGE and western blot. For total cell lysis (TCL), cells were lysed in $50 \mathrm{mM}$ Tris $\mathrm{pH} 7.5,150 \mathrm{mM} \mathrm{NaCl}, 1 \mathrm{mM} \mathrm{MgCl}_{2}, 1 \% \mathrm{SDS}$. TCL buffer was supplemented with Complete ${ }^{\circledR}$ protease inhibitor (Roche), phosphatase inhibitor cocktail (Roche) and Benzonase (VWR/Fischer scientific) at $1 \mu \mathrm{l}$ per $\mathrm{ml}$ buffer. Samples were boiled in $3 \mathrm{x}$ Laemelli buffer prior to SDS-PAGE. Unless otherwise stated, NuPAGETM $4-12 \%$ Bis-Tris gradient gels (Invitrogen) were used. Gels were transferred onto activated PVDF membranes (Immobilon Psq, $0.2 \mu \mathrm{m}$, Merck) prior to blocking and incubation with the indicated primary antibodies.

Autophagy flux by flow cytometry assay. U2OS ATG2A/2B DKO cells were transfected with mCherry-EGFP-LC3B tandem tagged reporter construct [45, 54], grown in 
G418/Neomycin selection $(800 \mu \mathrm{g} / \mathrm{ml})$ and single cell cloned. U2OS-ATG2A/B DKO-Tandem LC3B cells were then transduced with retrovirus containing iTAP ATG2A constructs and selected for in G418 + 1.5ug/ml puromycin and stable cells generated. These were treated as indicated, scraped in PBS and fixed in 4\% PFA for 15mins, washed and then subjected to flow cytometry analysis. All flow cytometry experiments were at least three times using 10,000 cells per cell line per treatment. The cells were then analysed and sorted on an LSR Fortessa (Becton Dickinson) flow cytometer. cells were gated according to forward scatter and side scatter and dead cells were excluded from analysis. GFP fluorescence measured by excitation at $488 \mathrm{~nm}$ and emission detected at $530 \pm 30 \mathrm{~nm}$ and mCherry fluorescence measured by excitation at $561 \mathrm{~nm}$ and emission detected at $610 \pm 20 \mathrm{~nm}$. Flow data was analysed using FlowJo software.

Immunofluorescence \& confocal microscopy. Cells grown $18 \mathrm{~mm}$ glass coverslips, were treated as described and subsequently fixed in $4 \%$ paraformaldehyde/PBS (PFA; Santa Cruz, 30525-89-4) for $10 \mathrm{~min}$ at room temperature and washed 3x in PBS. Cells were then washed in PBS $/ 0.1 \%$ saponin twice and primary antibodies incubated for $1 \mathrm{hr}$ at room temperature in 5\% BSA/PBS/0.1\% saponin. DAPI (Molecular Probes) was added during primary antibody incubation. Coverslips were then washed twice in PBS $/ 0.1 \%$ saponin and secondary antibodies (Invitrogen Donkey Anti-Mouse, -Rabbit, -Rat Alexa dyes (488, 555, 647 ) were used in combination depending on the primary antibody species and incubated in $\mathrm{PBS} / 5 \% \mathrm{BSA} / 0.1 \%$ saponin. For detection of endogenous GFP-ATG2A, nanobody boosters towards GFP (anti-GFP, Atto-488 coupled, Chromotek; gba488-100) were used to enhance the signal. Secondary antibodies were then washed twice in $\mathrm{PBS} / 0.1 \%$ saponin, once in PBS and once in $\mathrm{ddH}_{2} \mathrm{O}$ to remove the residual saponin prior to mounting in ProLong Diamond Antifade containing Mowiol (Invitrogen, p36965). Cells were imaged using a Zeiss 710 confocal microscope with a $63 \times$ objective lens. Subsequent image analysis was performed using FIJI (ImageJ) [55].

Lipid droplet induction and imaging. Cells were set up on glass coverslips and incubated with either complete medium plus $2 \%$ BSA or complete media plus $2 \%$ BSA $/ 500 \mu M$ oleic aid for $16 \mathrm{~h}$. Cells were then fixed in 4\% PFA/PBS for 10 minutes and permeabilised using the saponin method detailed above. Coverslips were incubated with anti-HA primary antibody and donkey anti-rat Alexa 657 secondary antibody. Lipid droplets were stained using $5 \mu \mathrm{M}$ BODIPY 493/503 (Thermo Fisher Scientific) to stain neutral lipids. Samples were mounted, imaged and analysed as detailed above.

Protein expression and purification. GST-tagged mammalian ATG8 fusion proteins were cloned into pGEX-4T-1 (GE Healthcare) and expressed in Escherichia coli BL21 (DE3) cells in LB medium as previously described [5]. Expression was induced by addition of $0.5 \mathrm{mM}$ 
IPTG and cells were incubated at $16^{\circ} \mathrm{C}$ overnight. Harvested cells were lysed using sonication in a lysis buffer $(20 \mathrm{mM}$ Tris- $\mathrm{HCl}$ pH 7.5, $10 \mathrm{mM}$ EDTA, $5 \mathrm{mM}$ EGTA, 150mM $\mathrm{NaCl}$ ) and the supernatant was subsequently applied to Glutathione Sepharose 4B beads (GE Healthcare). After several washes, fusion protein-bound beads were used directly in GST pulldown assays.

Proteinase $\mathrm{K}$ protection assay. Proteinase $\mathrm{K}$ assay was performed as previously detailed[54]. Briefly, U2OS ATG2A/B DKO or DKO plus ATG2A-WT, ATG2A-mLIR or ATG2A-mYFS cells were grown in complete media or starved (EBSS) in the presence of BafilomycinA1 (200nM) for $4 \mathrm{~h}$, scraped in PBS and centrifuged at $500 \mathrm{x}$. The cells were then resuspended in PBS $/ 6.5 \mu \mathrm{g} / \mathrm{ml}$ digitonin incubated for $5 \mathrm{~min}$ at room temperature and then for a further $30 \mathrm{~min}$ on ice. Samples were subsequently centrifuged at $13,000 \mathrm{xg}$ and the supernatant removed. The membrane fractions were then resuspended in $50 \mathrm{mM}$ Tris, $\mathrm{pH} 7.5,0.18 \mathrm{M}$ Sucrose. Resuspended membrane pellets were then incubated with either buffer only, buffer $+100 \mathrm{ng} / \mathrm{ml}$ proteinase $\mathrm{K}(\mathrm{PK})$ or $\mathrm{PK}+0.1 \%$ Triton $\mathrm{X}-100(\mathrm{PK}+\mathrm{TX})$ for $10 \mathrm{~min}$ at $30^{\circ} \mathrm{C}$. The reaction was stopped by addition of $3 \mathrm{x}$ Laemmli sample buffer and boiled at $95^{\circ} \mathrm{C}$.

Cloning and plasmid generation. pDONOR-ATG2A and pDONOR-ATG2B were kind gifts from C.Behrends. These were used in conjunction with Gateway cloning system (Invitrogen) pDEST-CMV-Myc and pMSCV-Flag-HA-IRES-Puro (iTAP) to generate plasmids expressing either ATG2A or ATG2B. Site-directed mutagenesis was carried out to mutate the wild-type gene for the required amino acid substitutions.

\section{Plasmids}

\begin{tabular}{|l|l|l|}
\hline \multicolumn{1}{|c|}{ Plasmid/epitope tag } & \multicolumn{1}{|c|}{ Gene/mutation } & Reference \\
\hline pDONOR223-hATG2A & Human ATG2A & This study \\
\hline pDONOR223-hATG2B & Human ATG2B & This study \\
\hline (iTAP) pMSCV-Flag-HA-ATG2A & ATG2A-WT & This study \\
\hline (iTAP) pMSCV-Flag-HA-ATG2A-mLIR & ATG2A aa1362-1365 FCIL/AAAA & This study \\
\hline (iTAP) pMSCV-Flag-HA-ATG2A-mYFS & ATG2A aa1395-1397 YFS/AAA & This study \\
\hline pDEST-CMV-Myc-ATG2A-WT & ATG2A-WT & This study \\
\hline pDEST-CMV-Myc-ATG2A-mLIR\#1 & ATG2A aa536-539 YTEI/AAAA & This study \\
\hline pDEST-CMV-Myc-ATG2A-mLIR\#2 & ATG2A aa926-929 FSTL/AAAA & This study \\
\hline pDEST-CMV-Myc-ATG2A-mLIR\#3 & ATG2A aa981-984 YFCL/AAAA & This study \\
\hline
\end{tabular}




\begin{tabular}{|c|c|c|}
\hline pDEST-CMV-Myc-ATG2A-mLIR\#4 & ATG2A aa1092-1095FLDV/AAAA & This study \\
\hline pDEST-CMV-Myc-ATG2A-mLIR \#5 & ATG2A aa1362-1365 FCIL/AAAA & This study \\
\hline pDEST-CMV-Myc-ATG2B-WT & ATG2B-WT & This study \\
\hline pDEST-CMV-Myc-ATG2B-mLIR\#1 & ATG2B aa845-848 WPRI/AAAA & This study \\
\hline pDEST-CMV-Myc-ATG2B-mLIR\#3 & ATG2B aa979-982 FENI/AAAA & This study \\
\hline pDEST-CMV-Myc-ATG2B-mLIR\#4 & $\begin{array}{l}\text { ATG2B aa1026-1029 } \\
\text { FSTV/AAAA }\end{array}$ & This study \\
\hline pDEST-CMV-Myc-ATG2B-mLIR\#5 & $\begin{array}{l}\text { ATG2B aa1306-1309 } \\
\text { YVRV/AAAA }\end{array}$ & This study \\
\hline pDEST-CMV-Myc-ATG2B-mLIR\#6 & ATG2B aa1491-1494 FCIL/AAAA & This study \\
\hline pGEX-4T1 alone & GST only & [5] \\
\hline pGEX-4T1-LC3A- $\Delta \mathrm{G}$ & MAP1LC3A deletion of c-term Gly & [5] \\
\hline pGEX-4T1-LC3B- $\triangle \mathrm{G}$ & MAP1LC3B deletion of c-term Gly & [5] \\
\hline pGEX-4T1-LC3C- $\triangle \mathrm{G}$ & MAP1LC3C deletion of c-term Gly & [5] \\
\hline pGEX-4T1-GABARAP- $\triangle \mathrm{G}$ & GABARAP deletion of c-term Gly & [5] \\
\hline pGEX-4T1-GABARAP-L1- $\triangle \mathrm{G}$ & $\begin{array}{l}\text { GABARAP-L1 deletion of c-term } \\
\text { Gly }\end{array}$ & [5] \\
\hline pGEX-4T1-GABARAP L2- $\Delta \mathrm{G}$ & $\begin{array}{l}\text { GABARAP-L2 deletion of c-term } \\
\text { Gly }\end{array}$ & [5] \\
\hline pEGFP-C1 empty & GFP only & [5] \\
\hline pEGFP-C1-LC3B & GFP-tagged MAP1LC3B & [5] \\
\hline pEGFP-C1-GABARAP & GFP-tagged GABARAP & [5] \\
\hline pMRXIP-GFP-Stx17 WT & GFP tagged Syntaxin 17 & [48] \\
\hline
\end{tabular}

Acknowledgements. We would like to acknowledge P.Crocker, I. Ganley, A.Gubas, I.Dikic and K.Ryan for critical reading of the manuscript and valuable insights and discussions. We are grateful to Prof. Sharon Tooze for providing a kind gift of WIPI2 and WIPI4 antibodies as well as WIPI expression plasmids. The anti-LAMP-2 (H4B4) and anti-c-Myc (9E10) 
antibodies were obtained from the Developmental Studies Hybridoma Bank, created by the NICHD of the NIH and maintained at The University of lowa. We gratefully acknowledge the support from ERASMUS+ for the support of MB and LvdB. This work was supported by a grant from Tenovus Scotland (T16/44).

Author contributions. MB, LVDB, NG and ND performed experiments, TJM designed and synthesised CRISPR/Cas9 guides, ARP assisted in confocal imaging and DGM designed, performed and analysed experiments. DGM wrote the manuscript.

Conflict of interest. The authors declare that they have no conflict of interest. 


\section{References:}

1. Galluzzi L, Baehrecke EH, Ballabio A, Boya P, Bravo-San Pedro JM, Cecconi F, Choi AM, Chu CT, Codogno P, Colombo Ml, et al. (2017) Molecular definitions of autophagy and related processes. The EMBO journal 36: 1811-1836

2. Novak I, Kirkin V, McEwan DG, Zhang J, Wild P, Rozenknop A, Rogov V, Lohr F, Popovic D, Occhipinti A, et al. (2010) Nix is a selective autophagy receptor for mitochondrial clearance. EMBO Rep 11: 45-51

3. McWilliams TG, Prescott AR, Allen GF, Tamjar J, Munson MJ, Thomson C, Muqit MM, Ganley IG (2016) mito-QC illuminates mitophagy and mitochondrial architecture in vivo. $J$ Cell Biol 214: 333-45

4. Wild P, Farhan H, McEwan DG, Wagner S, Rogov VV, Brady NR, Richter B, Korac J, Waidmann O, Choudhary C, et al. (2011) Phosphorylation of the autophagy receptor optineurin restricts Salmonella growth. Science 333: 228-33

5. Kirkin V, Lamark T, Sou YS, Bjorkoy G, Nunn JL, Bruun JA, Shvets E, McEwan DG, Clausen TH, Wild P, et al. (2009) A role for NBR1 in autophagosomal degradation of ubiquitinated substrates. Mol Cell 33: 505-16

6. Ganley IG, Lam du H, Wang J, Ding X, Chen S, Jiang X (2009) ULK1.ATG13.FIP200 complex mediates mTOR signaling and is essential for autophagy. The Journal of biological chemistry 284: 12297-305

7. Matsunaga K, Saitoh T, Tabata K, Omori H, Satoh T, Kurotori N, Maejima I, Shirahama-Noda K, Ichimura T, Isobe T, et al. (2009) Two Beclin 1-binding proteins, Atg14L and Rubicon, reciprocally regulate autophagy at different stages. Nat Cell Biol 11: 385-96

8. Zhong Y, Wang QJ, Li X, Yan Y, Backer JM, Chait BT, Heintz N, Yue Z (2009) Distinct regulation of autophagic activity by Atg14L and Rubicon associated with Beclin 1phosphatidylinositol-3-kinase complex. Nat Cell Biol 11: 468-76

9. Hanada T, Noda NN, Satomi Y, Ichimura Y, Fujioka Y, Takao T, Inagaki F, Ohsumi Y (2007) The Atg12-Atg5 conjugate has a novel E3-like activity for protein lipidation in autophagy. The Journal of biological chemistry 282: 37298-302

10. Pankiv S, Clausen TH, Lamark T, Brech A, Bruun JA, Outzen H, Overvatn A, Bjorkoy G, Johansen T (2007) p62/SQSTM1 binds directly to Atg8/LC3 to facilitate degradation of ubiquitinated protein aggregates by autophagy. The Journal of biological chemistry 282: 24131-45

11. Rogov V, Dotsch V, Johansen T, Kirkin V (2014) Interactions between autophagy receptors and ubiquitin-like proteins form the molecular basis for selective autophagy. Mol Cell 53: 167-78

12. Stolz A, Ernst A, Dikic I (2014) Cargo recognition and trafficking in selective autophagy. Nat Cell Biol 16: 495-501

13. Rogov VV, Stolz A, Ravichandran AC, Rios-Szwed DO, Suzuki H, Kniss A, Lohr F, Wakatsuki S, Dotsch V, Dikic I, et al. (2017) Structural and functional analysis of the GABARAP interaction motif (GIM). EMBO Rep 18: 1382-1396

14. Alemu EA, Lamark $T$, Torgersen KM, Birgisdottir $A B$, Larsen $K B$, Jain A, Olsvik $H$, Overvatn A, Kirkin V, Johansen T (2012) ATG8 family proteins act as scaffolds for assembly of the ULK complex: sequence requirements for LC3-interacting region (LIR) motifs. The Journal of biological chemistry 287: 39275-90

15. Lystad AH, Ichimura Y, Takagi K, Yang Y, Pankiv S, Kanegae Y, Kageyama S, Suzuki M, Saito I, Mizushima T, et al. (2014) Structural determinants in GABARAP required for the selective binding and recruitment of ALFY to LC3B-positive structures. EMBO Rep 15: 557-65

16. Olsvik HL, Lamark T, Takagi K, Larsen KB, Evjen G, Overvatn A, Mizushima T, Johansen T (2015) FYCO1 Contains a C-terminally Extended, LC3A/B-preferring LC3interacting Region (LIR) Motif Required for Efficient Maturation of Autophagosomes during Basal Autophagy. The Journal of biological chemistry 290: 29361-74

17. Zhou F, Wu Z, Zhao M, Murtazina R, Cai J, Zhang A, Li R, Sun D, Li W, Zhao L, et al. (2019) Rab5-dependent autophagosome closure by ESCRT. J Cell Biol 
18. Zhou F, Zou S, Chen Y, Lipatova Z, Sun D, Zhu X, Li R, Wu Z, You W, Cong X, et al. (2017) A Rab5 GTPase module is important for autophagosome closure. PLoS Genet 13: e1007020

19. Weidberg H, Shvets E, Shpilka T, Shimron F, Shinder V, Elazar Z (2010) LC3 and GATE-16/GABARAP subfamilies are both essential yet act differently in autophagosome biogenesis. The EMBO journal 29: 1792-802

20. Weidberg H, Shpilka T, Shvets E, Abada A, Shimron F, Elazar Z (2011) LC3 and GATE-16 $\mathrm{N}$ termini mediate membrane fusion processes required for autophagosome biogenesis. Dev Cell 20: 444-54

21. Nguyen TN, Padman BS, Usher J, Oorschot V, Ramm G, Lazarou M (2016) Atg8 family LC3/GABARAP proteins are crucial for autophagosome-lysosome fusion but not autophagosome formation during PINK1/Parkin mitophagy and starvation. J Cell Biol 215: 857-874

22. Fujita $\mathrm{N}$, Hayashi-Nishino $\mathrm{M}$, Fukumoto $\mathrm{H}$, Omori $\mathrm{H}$, Yamamoto $\mathrm{A}$, Noda $\mathrm{T}$, Yoshimori T (2008) An Atg4B mutant hampers the lipidation of LC3 paralogues and causes defects in autophagosome closure. Mol Biol Cell 19: 4651-9

23. Takahashi Y, He H, Tang Z, Hattori T, Liu Y, Young MM, Serfass JM, Chen L, Gebru M, Chen C, et al. (2018) An autophagy assay reveals the ESCRT-III component CHMP2A as a regulator of phagophore closure. Nat Commun 9: 2855

24. Romanyuk D, Polak A, Maleszewska A, Sienko M, Grynberg M, Zoladek T (2011) Human hAtg2A protein expressed in yeast is recruited to preautophagosomal structure but does not complement autophagy defects of atg2Delta strain. Acta Biochim Pol 58: 365-74

25. Kotani T, Kirisako H, Koizumi M, Ohsumi Y, Nakatogawa H (2018) The Atg2-Atg18 complex tethers pre-autophagosomal membranes to the endoplasmic reticulum for autophagosome formation. Proceedings of the National Academy of Sciences of the United States of America 115: 10363-10368

26. Obara K, Sekito T, Niimi K, Ohsumi Y (2008) The Atg18-Atg2 complex is recruited to autophagic membranes via phosphatidylinositol 3-phosphate and exerts an essential function. The Journal of biological chemistry 283: $23972-80$

27. Proikas-Cezanne T, Waddell S, Gaugel A, Frickey T, Lupas A, Nordheim A (2004) WIPI-1alpha (WIPI49), a member of the novel 7-bladed WIPI protein family, is aberrantly expressed in human cancer and is linked to starvation-induced autophagy. Oncogene 23: 9314-25

28. Polson HE, de Lartigue J, Rigden DJ, Reedijk M, Urbe S, Clague MJ, Tooze SA (2010) Mammalian Atg18 (WIPI2) localizes to omegasome-anchored phagophores and positively regulates LC3 lipidation. Autophagy 6: 506-22

29. Bakula D, Muller AJ, Zuleger T, Takacs Z, Franz-Wachtel M, Thost AK, Brigger D, Tschan MP, Frickey T, Robenek H, et al. (2017) WIPI3 and WIPI4 beta-propellers are scaffolds for LKB1-AMPK-TSC signalling circuits in the control of autophagy. Nat Commun 8: 15637

30. Zheng JX, Li Y, Ding YH, Liu JJ, Zhang MJ, Dong MQ, Wang HW, Yu L (2017) Architecture of the ATG2B-WDR45 complex and an aromatic Y/HF motif crucial for complex formation. Autophagy 13: 1870-1883

31. Chowdhury S, Otomo C, Leitner A, Ohashi K, Aebersold R, Lander GC, Otomo T (2018) Insights into autophagosome biogenesis from structural and biochemical analyses of the ATG2A-WIPI4 complex. Proceedings of the National Academy of Sciences of the United States of America 115: E9792-E9801

32. Kishi-Itakura C, Koyama-Honda I, Itakura E, Mizushima N (2014) Ultrastructural analysis of autophagosome organization using mammalian autophagy-deficient cells. $J$ Cell Sci 127: 4089-102

33. Velikkakath AK, Nishimura T, Oita E, Ishihara N, Mizushima N (2012) Mammalian Atg2 proteins are essential for autophagosome formation and important for regulation of size and distribution of lipid droplets. Mol Biol Cell 23: 896-909

34. Noda NN, Ohsumi Y, Inagaki F (2010) Atg8-family interacting motif crucial for selective autophagy. FEBS Lett 584: 1379-85 
35. Johansen T, Lamark T (2011) Selective autophagy mediated by autophagic adapter proteins. Autophagy 7: 279-96

36. Rozenknop A, Rogov VV, Rogova NY, Lohr F, Guntert P, Dikic I, Dotsch V (2011) Characterization of the interaction of GABARAPL-1 with the LIR motif of NBR1. J Mol Biol 410: $477-87$

37. Wu F, Watanabe Y, Guo XY, Qi X, Wang P, Zhao HY, Wang Z, Fujioka Y, Zhang H, Ren JQ, et al. (2015) Structural Basis of the Differential Function of the Two C. elegans Atg8 Homologs, LGG-1 and LGG-2, in Autophagy. Mol Cell 60: 914-29

38. Behrends C, Sowa ME, Gygi SP, Harper JW (2010) Network organization of the human autophagy system. Nature 466: 68-76

39. Kalvari I, Tsompanis S, Mulakkal NC, Osgood R, Johansen T, Nezis IP, Promponas VJ (2014) iLIR: A web resource for prediction of Atg8-family interacting proteins. Autophagy 10: $913-25$

40. Popelka H, Klionsky DJ (2015) Analysis of the native conformation of the LIR/AIM motif in the Atg8/LC3/GABARAP-binding proteins. Autophagy 11: 2153-9

41. Jacomin AC, Samavedam S, Charles H, Nezis IP (2017) iLIR@viral: a web resource for LIR motif-containing proteins in viruses. Autophagy: 0

42. Sudhakar P, Jacomin AC, Hautefort I, Samavedam S, Fatemian K, Ari E, Gul L, Demeter A, Jones E, Korcsmaros T, et al. (2019) Targeted interplay between bacterial pathogens and host autophagy. Autophagy: 1-14

43. Birgisdottir AB, Mouilleron S, Bhujabal Z, Wirth M, Sjottem E, Evjen G, Zhang W, Lee R, O'Reilly N, Tooze SA, et al. (2019) Members of the autophagy class III phosphatidylinositol 3-kinase complex I interact with GABARAP and GABARAPL1 via LIR motifs. Autophagy: 1-23

44. Jatana N, Ascher DB, Pires DEV, Gokhale RS, Thukral L (2019) Human LC3 and GABARAP subfamily members achieve functional specificity via specific structural modulations. Autophagy

45. Kimura S, Noda T, Yoshimori T (2007) Dissection of the autophagosome maturation process by a novel reporter protein, tandem fluorescent-tagged LC3. Autophagy 3: 452-60

46. Tamura N, Nishimura T, Sakamaki Y, Koyama-Honda I, Yamamoto H, Mizushima N (2017) Differential requirement for ATG2A domains for localization to autophagic membranes and lipid droplets. FEBS Lett 591: 3819-3830

47. Klionsky DJ, Abdelmohsen K, Abe A, Abedin MJ, Abeliovich $\mathrm{H}$, Acevedo Arozena A, Adachi H, Adams CM, Adams PD, Adeli K, et al. (2016) Guidelines for the use and interpretation of assays for monitoring autophagy (3rd edition). Autophagy 12: 1-222

48. Itakura E, Kishi-Itakura C, Mizushima N (2012) The hairpin-type tail-anchored SNARE syntaxin 17 targets to autophagosomes for fusion with endosomes/lysosomes. Cell 151: $1256-69$

49. Stanga D, Zhao Q, Milev MP, Saint-Dic D, Jimenez-Mallebrera C, Sacher M (2019) TRAPPC11 functions in autophagy by recruiting ATG2B-WIPI4/WDR45 to preautophagosomal membranes. Traffic

50. Osawa T, Kotani T, Kawaoka T, Hirata E, Suzuki K, Nakatogawa H, Ohsumi Y, Noda NN (2019) Atg2 mediates direct lipid transfer between membranes for autophagosome formation. Nat Struct Mol Biol 26: 281-288

51. Valverde DP, Yu S, Boggavarapu V, Kumar N, Lees JA, Walz T, Reinisch KM, Melia TJ (2019) ATG2 transports lipids to promote autophagosome biogenesis. J Cell Biol

52. Kumar N, Leonzino M, Hancock-Cerutti W, Horenkamp FA, Li P, Lees JA, Wheeler $\mathrm{H}$, Reinisch KM, De Camilli P (2018) VPS13A and VPS13C are lipid transport proteins differentially localized at ER contact sites. J Cell Biol

53. Ran FA, Hsu PD, Wright J, Agarwala V, Scott DA, Zhang F (2013) Genome engineering using the CRISPR-Cas9 system. Nat Protoc 8: 2281-2308

54. McEwan DG, Popovic D, Gubas A, Terawaki S, Suzuki H, Stadel D, Coxon FP, Miranda de Stegmann D, Bhogaraju S, Maddi K, et al. (2015) PLEKHM1 regulates autophagosome-lysosome fusion through HOPS complex and LC3/GABARAP proteins. Mol Cell 57: 39-54 
bioRxiv preprint doi: https://doi.org/10.1101/624627; this version posted May 3, 2019. The copyright holder for this preprint (which was not certified by peer review) is the author/funder, who has granted bioRxiv a license to display the preprint in perpetuity. It is made available under aCC-BY-NC-ND 4.0 International license.

55. Schindelin J, Arganda-Carreras I, Frise E, Kaynig V, Longair M, Pietzsch T, Preibisch S, Rueden C, Saalfeld S, Schmid B, et al. (2012) Fiji: an open-source platform for biologicalimage analysis. Nat Methods 9: 676-82 
Figure 1. CRISPR/Cas9 GFP-tagged ATG2A localizes to early autophagy membranes.

(A) U2OS cells modified to expressed endogenous GFP-tagged ATG2A (green) were grown in complete media (CM) or starved (EBSS) for $2 \mathrm{~h}$ before fixation and immunostaining with antibodies against ATG2B (red) and LC3B (magenta) and analysed by confocal microscopy. Arrows mark ATG2A/LC3B/ATG2B positive structures. Scale bar 10 $\mu$ m.

(B) Cells were treated as in (A) and stained with anti-WIPI2 (red) or (C) anti-ATG16L1 (red) or (D) GABARAP-L1 (red). Arrows mark structures of interest. Scale bar 10 $\mu \mathrm{m}$.

(E) U2OS WT or U2OS GFP-ATG2A knock-in (KI) cells were grown in CM or starvation media for $2 \mathrm{~h}$, lysed and incubated with anti-GFP nanobody beads coupled to agarose to immunoprecipitate (IP) GFP-ATG2A. IP samples and 2\% input lysates were run on 4$12 \%$ gradient gel and processed for western blotting. Anti-ATG2A, anti-WIPI4 and antiLC3B, anti-GABARAP (pan) were used to probe for the presence/absence of autophagy proteins in the immunoprecipitated samples. p-p70S6K (T389) was used as a marker for starved cells and total p70S6K as loading control.

Figure 2. ATG2A and ATG2B contain a highly conserved LC3 Interaction Region (LIR).

(A) Myc-tagged ATG2A-wild type (WT), ATG2A-mutant LIR (mLIR; FCIL/AAAA) (upper blots) or Myc-tagged ATG2B-WT or ATG2B-mLIR (FCIL/AAAA; lower blots) were over expressed in HEK293T cells, and lysates were incubated with purified GST alone or GST-tagged LC3A, -LC3B, LC3C, -GABARAP, -GABARAP-L1 or GABARAP-L2. Samples were spun, washed and blotted for the presence/absence of Myc tagged ATG2s using anti-Myc antibody. GST-proteins were visualised by Ponceau S staining of membranes.

(B) Myc-tagged ATG2A-wild type (WT) and ATG2A-mLIR (orange) were co-expressed with GFP-alone, GFP-LC3B or GFP-GABARAP in HEK293T cells, lysed and anti-GFP nanobodies coupled to agarose were used to immunoprecipitate GFP-tagged proteins. Samples were subjected to western blotting and probed for the presence of Myc-ATG2A in immunoprecipitated samples. Anti-p62/SQSTM1 was used as an internal control for the immunoprecipitated samples.

(C) As in (B) but using Myc-tagged ATG2B-WT or ATG2B-mLIR (orange) co-expressed with GFP-alone, GFP-LC3B or GFP-GABARAP. Samples were subjected to western blotting and probed for the presence of Myc-ATG2B in immunoprecipitated samples. Antip62/SQSTM1 was used as an internal control for the immunoprecipitated samples.

(D) Domain structure of ATG2A (green) and ATG2B (grey) proteins. Both ATG2s contain an $\mathrm{N}$-terminal VPS13/Chorein domain, ATG2 C-terminal autophagy domain (CAD motif) 
and ATG2 c-terminal domain. Position and sequence of the new ATG2 LIR motif that directs GABARAP interaction (orange) and the previously identified WIPI4 interaction motif (YFS; purple box). Approximately 30 amino acids separate these two motifs in human ATG2A and ATG2B. Multiple sequence alignment of multiple species using Jalview highlights the conservation of these regions. Abbreviations for species: Hs Homo sapien; Mm - Mus musculus; Rn - Rattus norvegicus; Pt - Pan troglodytes; Xt Xenopus tropicalis Dr - Danio rerio; Dm - Drosophila melanogaster; Ce Caenorhabditis elegans; Sp - Schizosaccharomyces pombe; Sc - Saccharomyces cerevisiae.

(E) HA-tagged ATG2A-WT, -mLIR (orange) and -mYFS (YFS/AAA; purple) were stably expressed in U2OS ATG2A/B double knock-out cells using retrovirus transduction. Cells were transfected with GFP-GABARAP and $24 \mathrm{~h}$ later grown in complete medium (CM) or starved for $2 \mathrm{~h}$ (EBSS). Cells were lysed and anti-HA beads were used to immunoprecipitate HA-tagged ATG2A and processed for western blot. Blots were then probed with antibodies against HA-tag (ATG2A), anti-WIPI4, and anti-GFP for the presence/absence in immunoprecipitated samples. All blots are representative of at least $\mathrm{n}=3$ independent experiments.

Figure 3. ATG2A LIR domain is essential for autophagy flux.

(A) U2OS ATG2A/B double knock-out (DKO) CRISPR/Cas9 cells stably expressing Tandem tagged LC3B (mCherry-GFP-LC3B) were retrovirally transduced to express vector, or HA-tagged ATG2A-WT, -mLIR (FCIL/AAAA) or -mYFS (YFS/AAA). Cells were grown in complete medium (CM) or starved for $2 \mathrm{~h}$ (EBSS) or treated with CM plus BafilomycinA1 (200nM, 4 h), fixed and analysed by confocal microscopy. Merged images of GFP (green) and mCherry (red) channels show the presence of autophagosomes/phagophores (GFP and mCherry positive, yellow puncta) or autolysosomes (mCherry only, red puncta) Scale bar 10 $\mu \mathrm{m}$. Images are representative of $n=3$ independent experiments.

(B) Quantification of (A) using flow cytometry of measuring GFP and mCherry fluorescence. Cells were gated based on GFP and mCherry fluorescence and \% mCherry positive cells gated and used as an indication of autolysosome formation due to GFP quenching. Each symbol represents an $n=1$ independent experiment with 10,000 cells analysed per condition. Horizontal bar indicates mean \pm SD.

(C) U2OS ATG2A/B DKO cells reconstituted with vector only, HA-ATG2A-WT, -mLIR and mYFS were stimulated with complete medium, (CM), $2 \mathrm{~h}$ starvation (EBSS) or $4 \mathrm{~h}$ BafilomycinA1 (BafA1, 200nM), lysed in total cell lysis buffer and subjected to western 
blot analysis. Blots were probed for the presence of HA-tag (ATG2A), p62/SQSTM1, LC3B and vinculin (loading control).

(D) p62/SQSTM1 and LC3B-II (E) levels were normalized to loading control and quantified as fold change of DKO proteins levels. Each symbol represents an independent experiment. Quantification of at least $\mathrm{n}=3$ independent experiments is shown. Horizontal bar represents mean $\pm S D$.

\section{Figure 4. Mutation of ATG2A LIR prevents phagophore closure.}

(A) Graphical representation of proteinase $\mathrm{K}$ assay (i) showing protection of p62/SQSTM1 inside sealed autophagosomes or proteinase $\mathrm{K}$ sensitive $\mathrm{p} 62$ present within open phagophores. (ii) Graphical representation of sytnatxin17 (STX17) translocation to completed autophagosomes and not phagophores. Autophagosomes are identified as being both LC3B and STX17 positive vesicles.

(B) U2OS ATG2A/B DKO cells reconstituted with vector only, HA-ATG2A-WT, -mLIR and mYFS were stimulated with complete medium, (CM), $4 \mathrm{~h}$ starvation (EBSS) plus BafilomycinA1 (BafA1, 200nM) treatment. Cells were centrifuged and resuspended in PBS digitonin, spun, washed and the membrane fractions incubated with proteinase $\mathrm{K}$ with and without $0.1 \%$ TritonX-100. Sampels were then subjected to western blotting using anti-p62/SQSTM1 and anti-HA (ATG2A) antibodies. Percentage p62/SQSTM1 remaining was calculated using densitometry analysis. Blots are representative of $n=3$ independent experiments.

(C) U2OS ATG2A/B DKO cells reconstituted with vector only, HA-ATG2A-WT, -mLIR and mYFS and stably expressing GFP-Syntaxin17 (STX17) were stimulated starvation (EBSS) plus BafilomycinA1 (BafA1, 200nM) for 4 hours to stimulate autophagosome generation and prevent their degradation in the lysosome. Cells were fixed and immunestained for LC3B (magenta). DAPI was included (blue) to mark the DNA/nucleus. Confocal analysis of LC3B and GFP-STX17 (green) localization was performed. Closed arrows (ATG2A/B DKO and DKO + ATG2A mLIR) highlight aggregate structures. Open arrows (DKO + ATG2A-WT and ATG2A-mYFS) highlight STX17/LC3B positive vesicles. Scale bar $10 \mu \mathrm{m}$.

(D) Quantification of (C) expressed as a percentage of cells with STX17/LC3B positive vesicles. Each symbol represents a single field of cells with 5-10 cells per field. A total of 300-600 cells were analysed over $n=3$ independent experiments.

(E) Model of ATG2 function based on the current knowledge. ATG2A localizes to ER membranes and facilitates lipid transfer from the ER to the growing phagophore. ATG2 
interaction with GABARAP is essential for anchoring ATG2 to growing phagophore and mutation of the GABARAP interaction region results in the formation of immature phagophores that fail to close.

Table 1. Potential LC3 interaction region sequences identified in human ATG2A and ATG2B protein sequences.

\begin{tabular}{|c|c|c|c|c|}
\hline $\begin{array}{c}\text { LIR } \\
\text { number }\end{array}$ & Gene & $\begin{array}{l}\text { Potential LIR } \\
\text { sequence }\end{array}$ & $\begin{array}{l}\text { Amino acid } \\
\text { position } \\
\text { (WxxL) }\end{array}$ & $\begin{array}{c}\text { Mutant LIR interaction with } \\
\text { GST-LC3/GABARAPs? }\end{array}$ \\
\hline 1 & ATG2A & GTSEPEYTEILT & $536-539$ & +++ \\
\hline 2 & ATG2A & SLHQSTFSTLVT & $926-929$ & - \\
\hline 3 & ATG2A & GQPGLGYFCLEA & $981-984$ & +++ \\
\hline 4 & ATG2A & HSQLLEFLDVLDD & $1092-1095$ & +++ \\
\hline 5 & ATG2A & TLDSDEFCILDAP & $1362-1365$ & - \\
\hline 1 & ATG2B & SSDDFDWPRIVL & $845-848$ & +++ \\
\hline 2 & ATG2B & LPNKSFYEKLYN & $954-957$ & Present on alpha helix \\
\hline 3 & ATG2B & PSPVETFENISY & $979-982$ & Not expressed \\
\hline 4 & ATG2B & EETLQYFSTVDP & $1026-1029$ & ++ \\
\hline 5 & ATG2B & INLSRDYVRVMD & $1306-1309$ & Not expressed \\
\hline 6 & ATG2B & PTENDDFCILFAP & $1491-1494$ & - \\
\hline
\end{tabular}




\section{Expanded View.}

\section{A conserved ATG2-GABARAP interaction is critical for phagophore closure.}

Mihaela Bozic ${ }^{1}$, Luuk van den Bekerom ${ }^{1}$, Beth A. Milne ${ }^{1}$, Nicola Goodman ${ }^{1}$, Lisa Roberston ${ }^{1}$, Alan R. Prescott ${ }^{2}$, Thomas J. Macartney ${ }^{3}$, Nina Dawe ${ }^{1} \&$ David G. McEwan ${ }^{1 *}$

4. Division of Cell Signalling \& Immunology, School of Life Sciences, University of Dundee, Dundee, Scotland, United Kingdom.

5. Dundee Imaging Facility, School of Life Sciences, University of Dundee, Dundee, UK.

6. MRC Protein Phosphorylation and Ubiquitylation Unit, School of Life Sciences, University of Dundee, Dow Street, Dundee DD1 5EH, UK

${ }^{*}$ Correspondence to: $\underline{\text { d.g.mcewan@dundee.ac.uk }}$ 
Figure EV1. Generation of GFP-tagged endogenous ATG2A.

(A) Strategy for insertion of GFP-tag upstream of human ATG2A exon 1. Graphic shows position of guides and locus before and after (B) GFP-tag plus linker insertion.

(C) Western blot of total cell lysates from parental wild type (WT) and GFP-ATG2A CRISPR/Cas9 Knock-in clones 1 and 2 using anti-ATG2A and Atnti-ATG2B antibodies.

(D) Myc-tagged ATG2A-wild type (WT) or ATG2B-WT (E) was co-expressed with GFPalone, GFP-LC3B or GFP-GABARAP in HEK293T cells, lysed and anti-GFP nanobodies used to immunoprecipitate GFP-tagged proteins. Samples were subjected to western blotting and probed for the presence of Myc-ATG2A in immunoprecipitated samples. Anti-p62/SQSTM1 was used as an internal control for the immunoprecipitated samples.

Figure EV2. ATG2A and ATG2B contain a highly conserved LC3 Interaction Region (LIR).

(A) Domain structure of ATG2A (green) and ATG2B (grey) proteins. Both ATG2s contain an $\mathrm{N}$-terminal VPS13/Chorein domain, ATG2 C-terminal autophagy domain (CAD motif) and ATG2 c-terminal domain. Position and sequence of putative ATG2 LC3 interaction regions (LIRs) as identified by iLIR and manual annotation. ATG2A has 5 potential LIRs and ATG2B has 6 potential LIRs. See Table 1 for details.

(B) Myc-tagged ATG2A-wild type (WT) and putative LIR mutants, where the potential core motif was mutated to alanine were used in a pull-down assay with GST-tagged mammalian ATG8 proteins. Shown are ATG2-mLIR\#1, ATG2-mLIR\#2 (C), ATG2mLIR\#3 (D) and ATG2-mLIR\#4 (E). ATG2-mLIR\#5 is shown on Figure 2A. ATG2AWT or -mLIRs were over expressed in HEK293T cells, and lysates were incubated with purified GST alone or GST-tagged LC3A, -LC3B, LC3C, -GABARAP, -GABARAP-L1 or GABARAP-L2. Samples were spun, washed and blotted for the presence/absence of Myc tagged ATG2A using anti-Myc antibody. Anti-p62/SQSTM1 was used as an internal control for the GST-pull-down samples GST-proteins were visualised by Ponceau $S$ staining of membranes.

(F) As in (B) but using Myc-tagged ATG2B-WT or ATG2B-mLIR proteins. Shown are ATG2B mLIR\#1 and ATG2B mLIR\#4 (G). ATG2B-mLIR\#2 was present on an alpha helix, whereas ATG2B-mLIR\#3 and ATG2B-mLIR\#5 were not expressed. ATG2B-mLIR\#6 is shown on Figure 2A. See also Table 1. Anti-p62/SQSTM1 was used as an internal control for the GST-pull-down samples. All blots are representative of at least $n=3$ independent experiments 
Figure EV3. ATG2A-WT and mutants effectively localize to lipid droplets.

(A) U2OS ATG2A/B DKO cells reconstituted with vector only, HA-ATG2A-WT, -mLIR and mYFS were stimulated starvation (EBSS) plus BafilomycinA1 (BafA1, 200nM) for 4 hours to stimulate autophagosome generation and prevent their degradation in the lysosome. Cells were fixed and immune-stained for LC3B (magenta) and LAMP2 to visualise lysosomes. DAPI was included (blue) to mark the DNA/nucleus. Closed arrows (ATG2A/B DKO and DKO + ATG2A mLIR) highlight aggregate structures. Open arrows (DKO + ATG2A-WT and ATG2A-mYFS) highlight LAMP2/LC3B positive vesicles. Scale bar $10 \mu \mathrm{m}$.

(B) U2OS ATG2A/B DKO cells reconstituted with vector only, HA-ATG2A-WT, -mLIR and mYFS were stimulated with either $2 \%$ BSA only or (C) $2 \% B S A$ plus $500 \mu \mathrm{M}$ oleic acid for $16 \mathrm{~h}$ prior to fixation in 4\% PFA. Cells were permeabilised using saponin and stained with anti-HA (ATG2A; magenta) and 5 $\mu$ M BODIPY 493/503 to visualise lipid droplets (green; LDs). DAPI was included (blue) to mark the DNA/nucleus. Arrows arrows (ATG2A/B DKO and DKO + ATG2A mLIR) highlight aggregate structures. Open arrows highlight ATG2A positive lipid droplets. All images are representative of at least $n=3$ independent experiments. Scale bar $10 \mu \mathrm{m}$.

Figure EV4. ATG2A-mLIR causes accumulation of early autophagy markers.

(A) U2OS ATG2A/B DKO cells reconstituted with vector only, HA-ATG2A-WT, -mLIR and mYFS were stimulated starvation (EBSS) 2 hours to stimulate autophagosome generation. Cells were then fixed and immune-stained for LC3B (green), p62/SQSTM1 (magenta) or (B) ATG9A, (C) WIPI2 and (D) ATG16L1 (Magenta). Anti-HA (ATG2A expression) (cyan) and DAPI (blue; DNA/nucleus) were included. Closed arrows (ATG2A/B DKO and DKO + ATG2A mLIR) highlight LC3B positive aggregate structures. Open arrows (DKO + ATG2A-WT and ATG2A-mYFS) highlight LC3B positive vesicles. All images are representative of at least $n=3$ independent experiments. Scale bar $10 \mu \mathrm{m}$. 


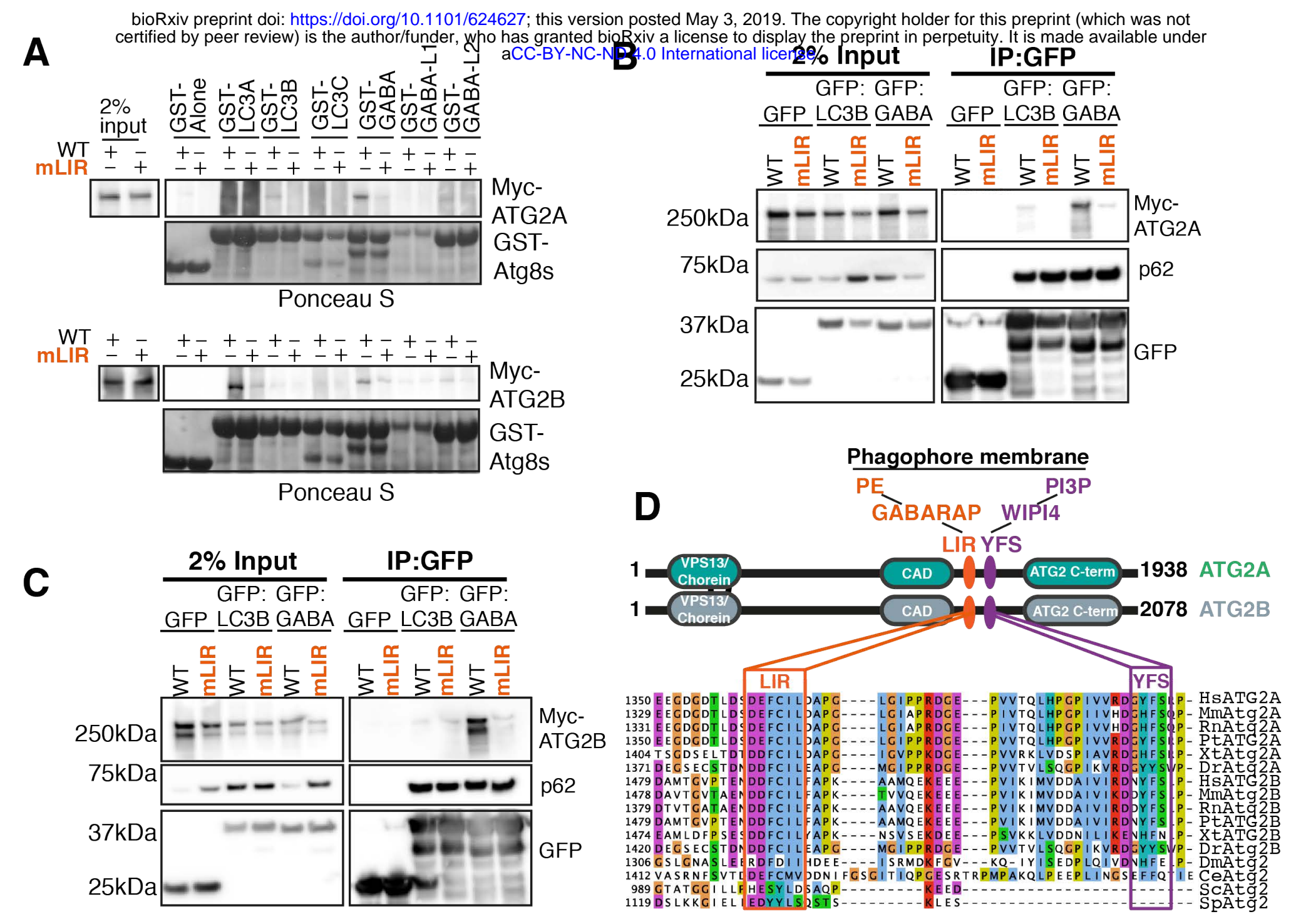

E

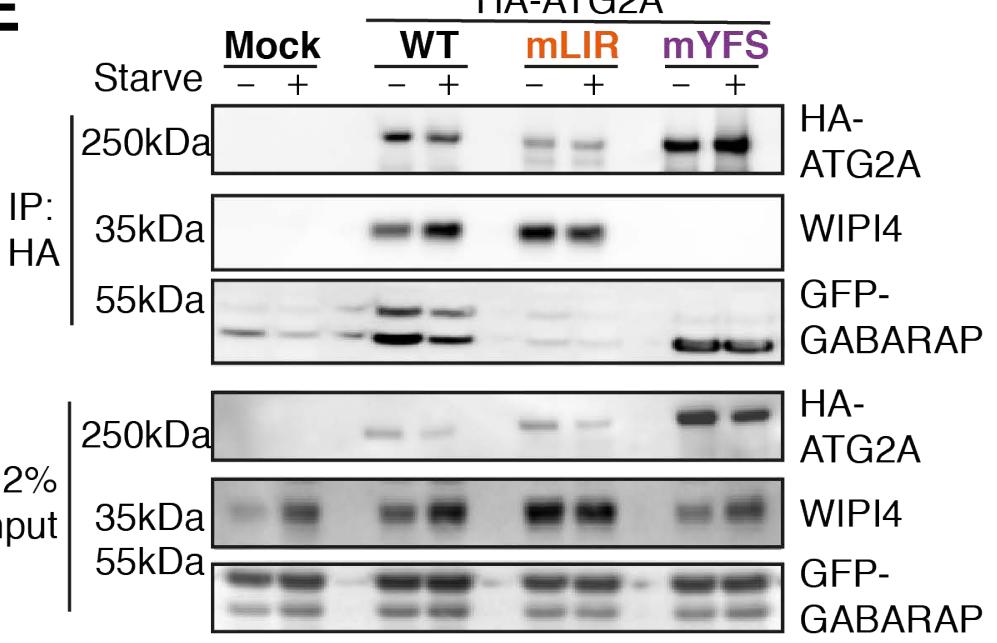


Figure 3

mCherry-GFP-LC3B
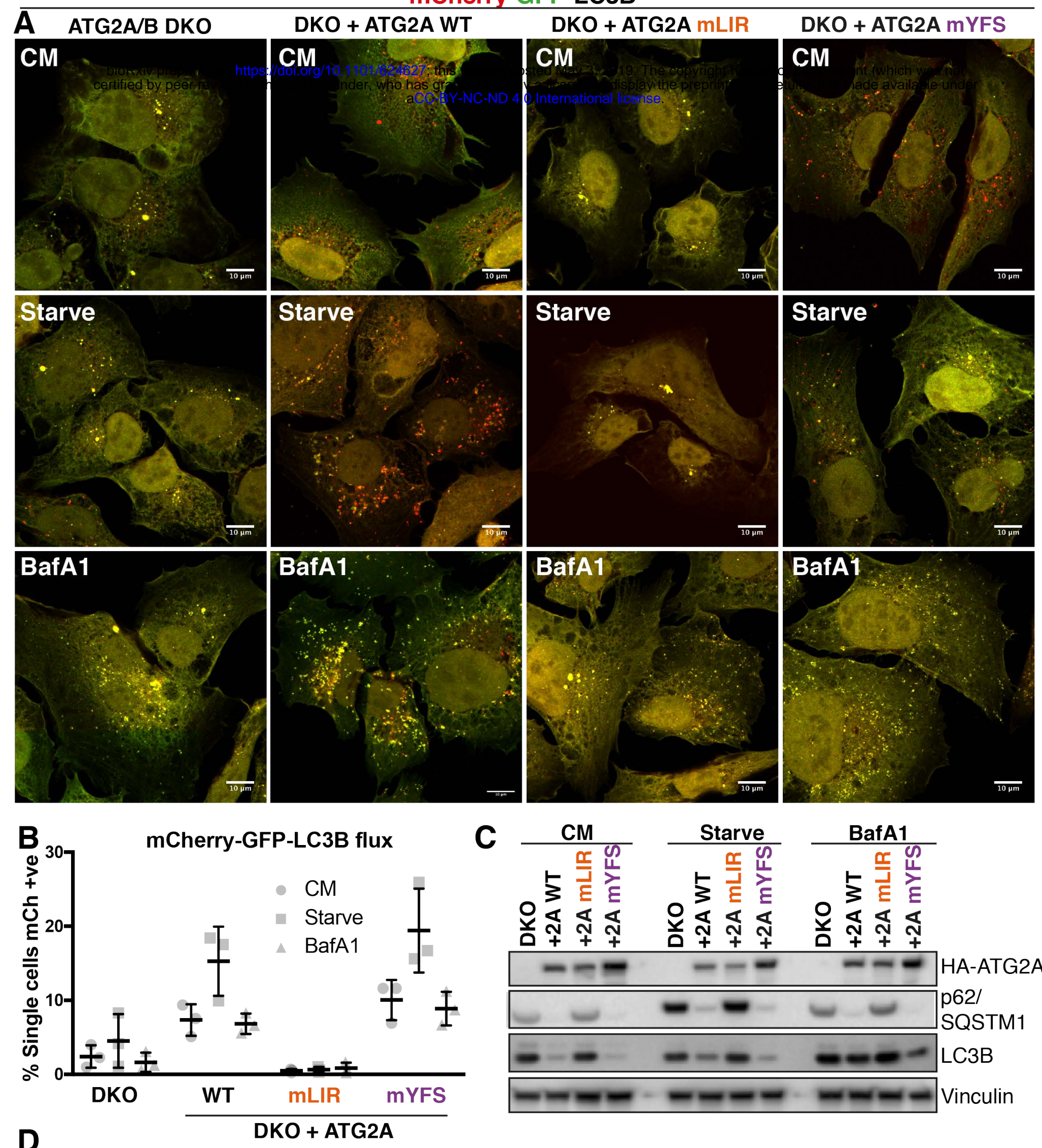

C $\frac{\mathrm{CM}}{\infty} \quad \frac{\text { Starve }}{\text { BafA1 }}$
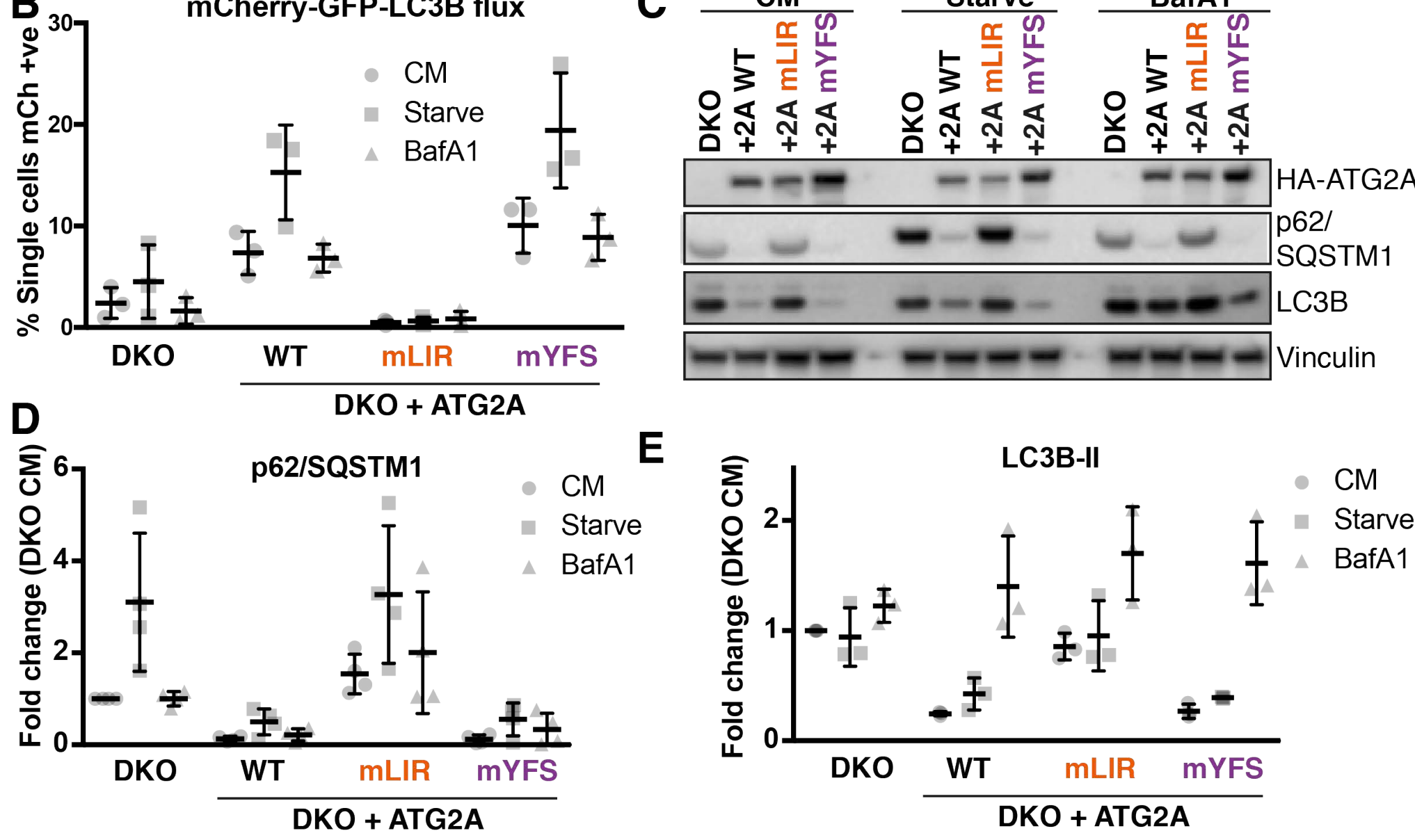
Figure 4

A Phagophore

Autophagoso

Protected

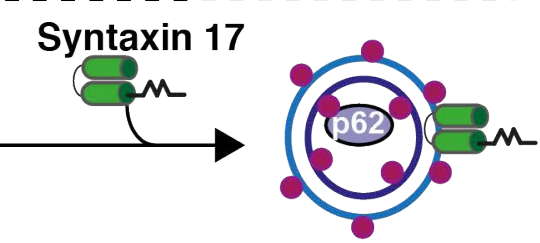

STXN17 +ve
Degraded

(ii)

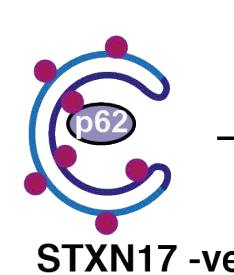

(i)

STXN17 -ve

\section{B ATG2A/B}

DKO + ATG2A

WT mLIR mYFS

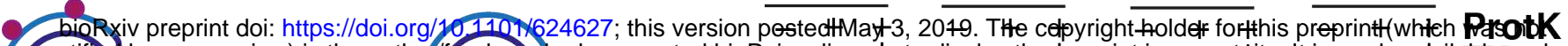

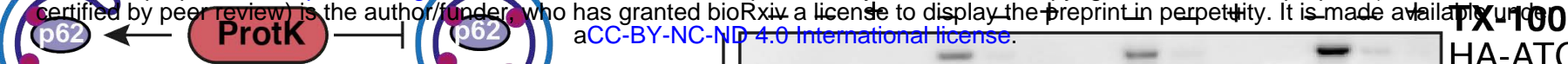
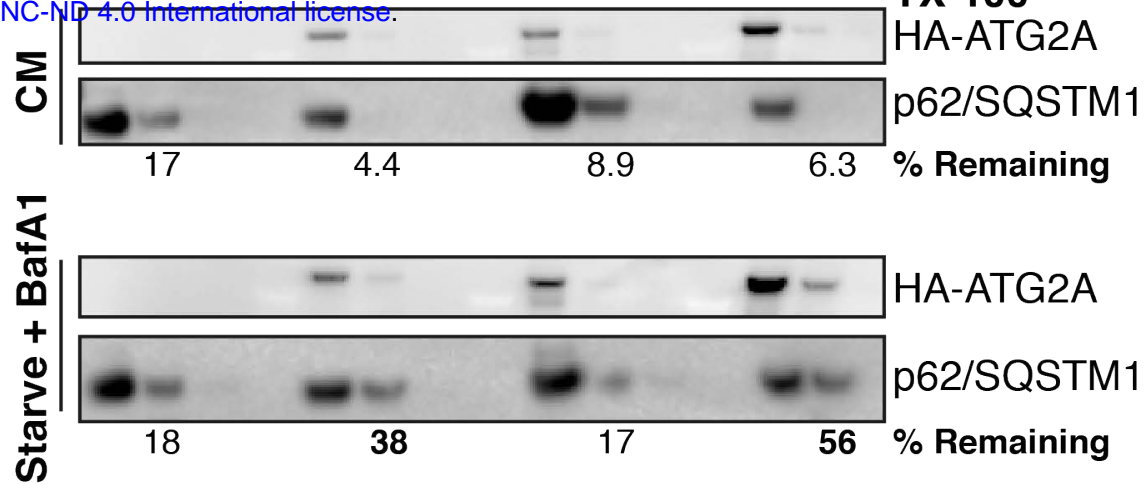

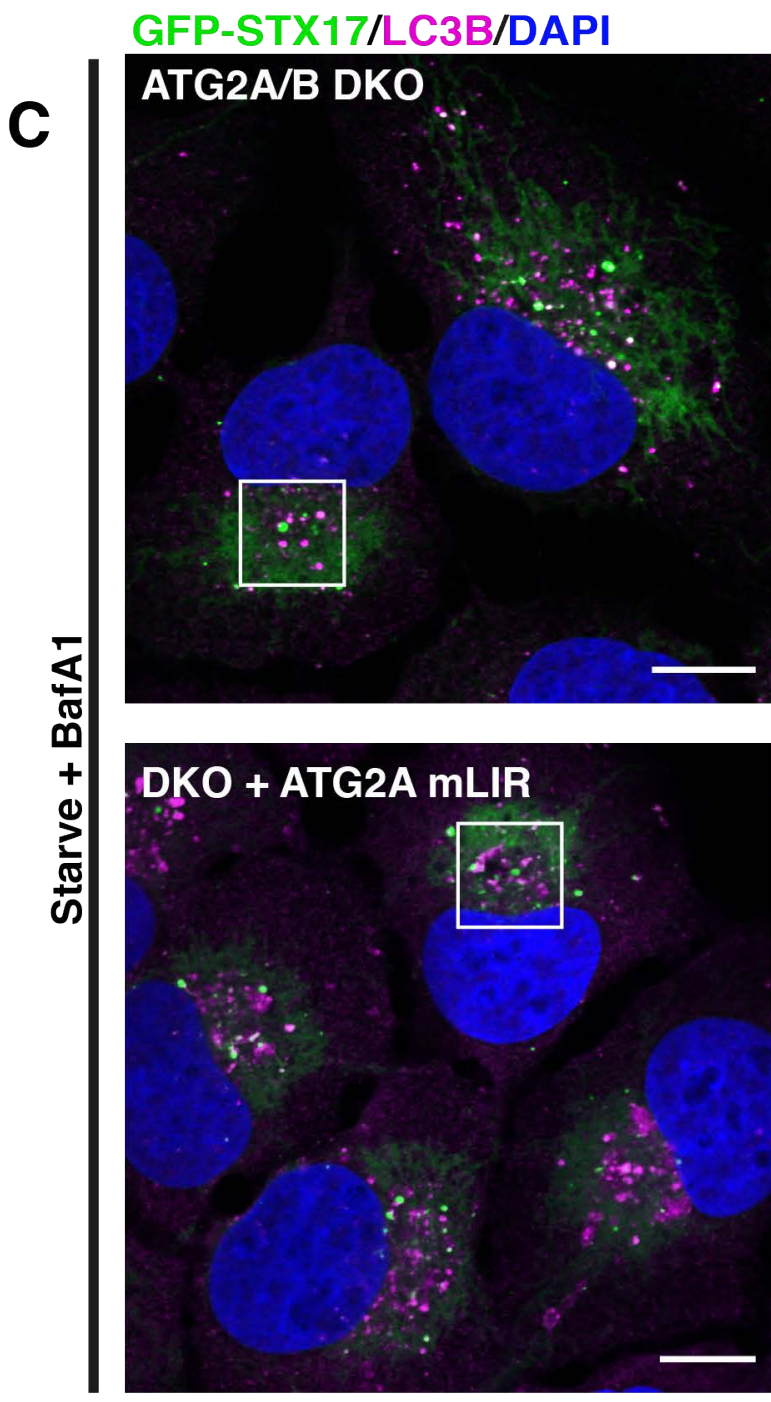

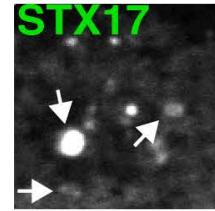
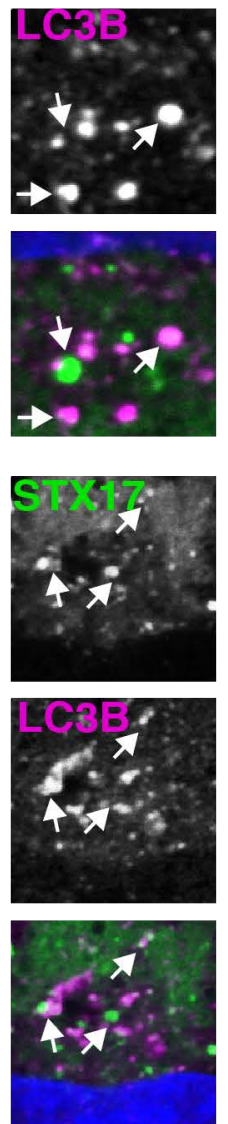

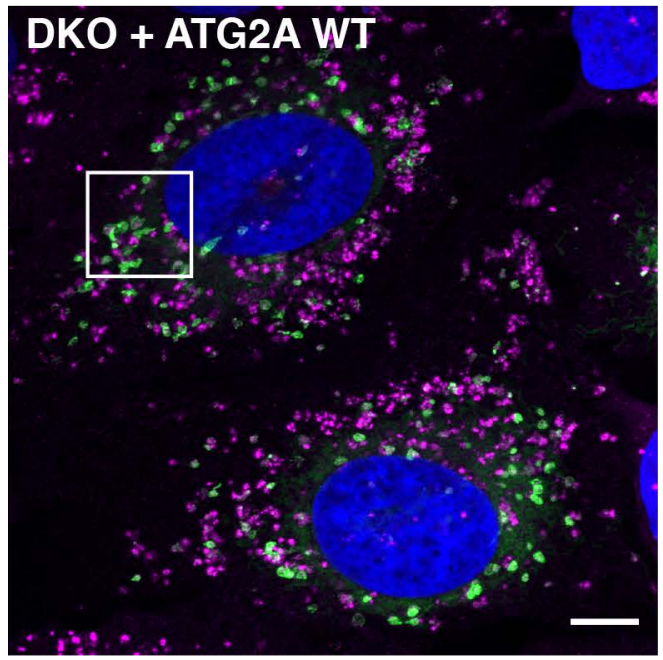

\section{DKO + ATG2A mYFS}
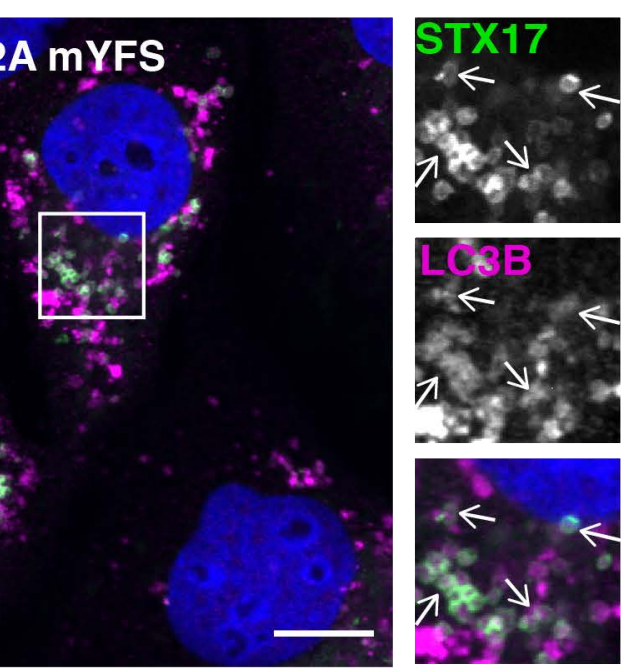

E Gabarap

WIPI4

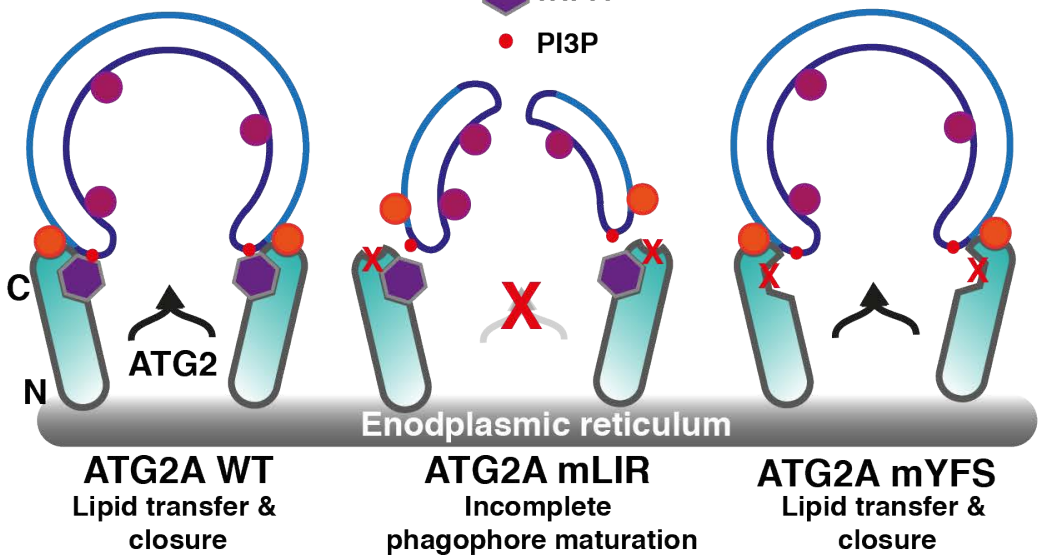

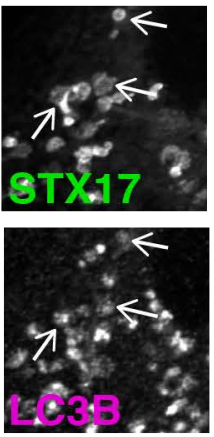

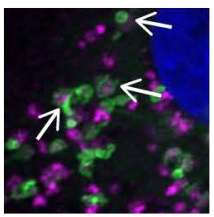

S

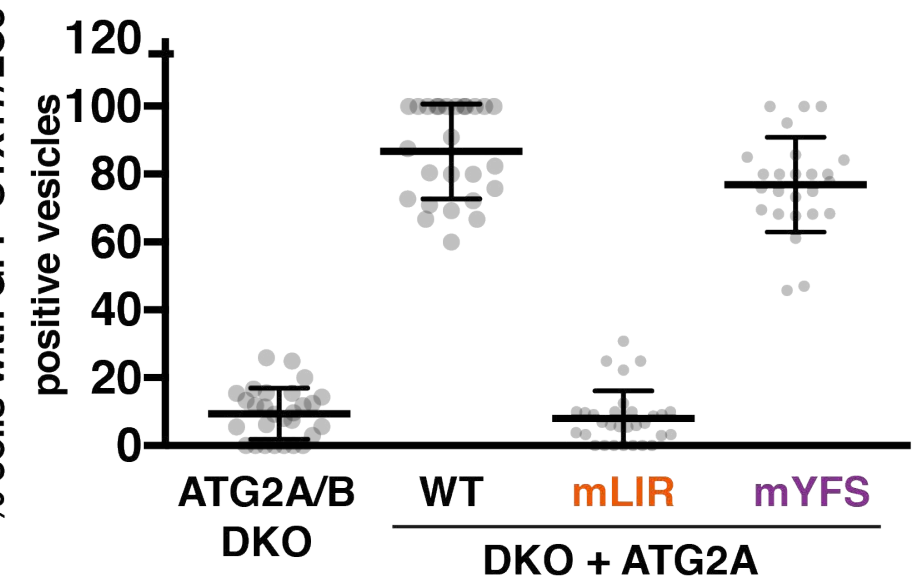


Figure EV1
\[ \begin{array}{l}\text { bioRxiv preprint doi: https://doi.org/10.1101/624627; this version posted May 3, 2019. The copyright holder for this preprint (which was not } \\ \text { certified by peer review) is the author/funder, who has granted bioRxiv a license to display the preprint in perpetuity. It is made available und } \\ \text { Human ACC-BY-NC-ND } 4.0 \text { International license. }\end{array} \]

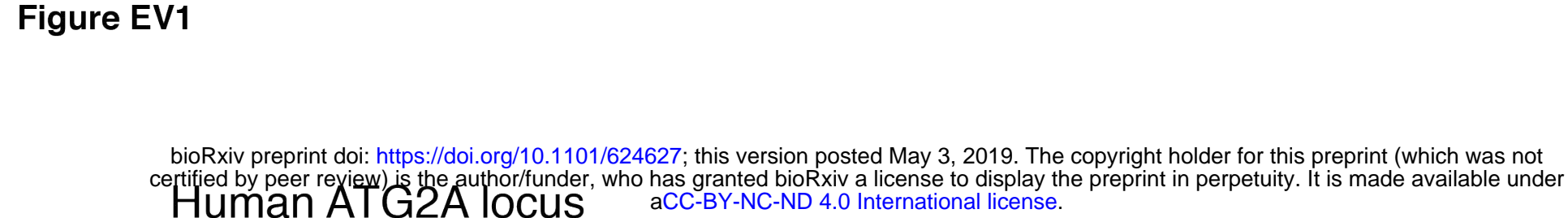

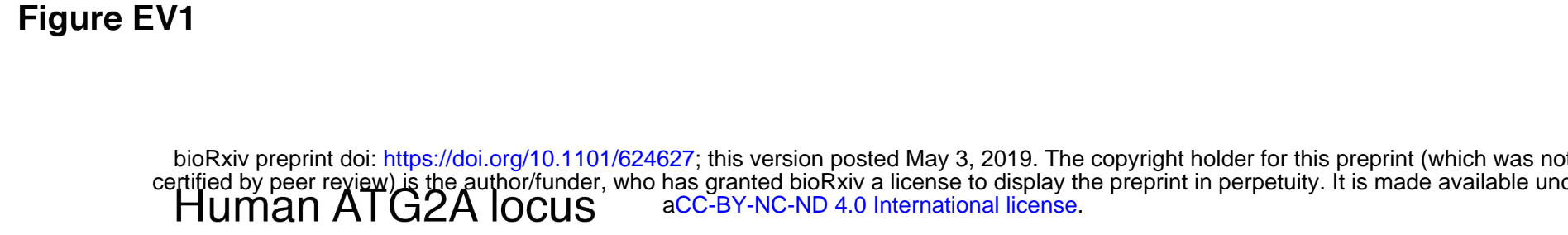

A

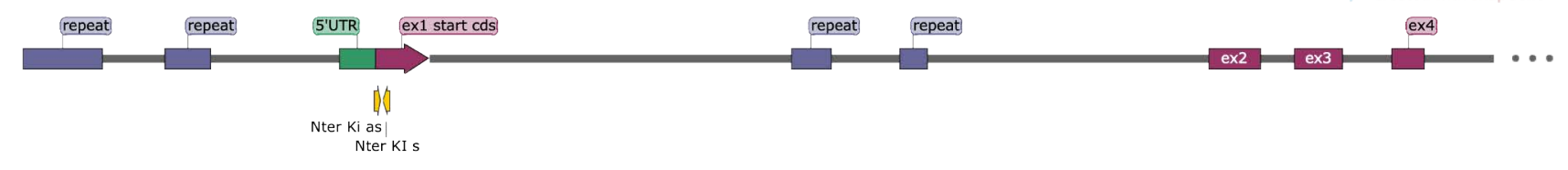

B

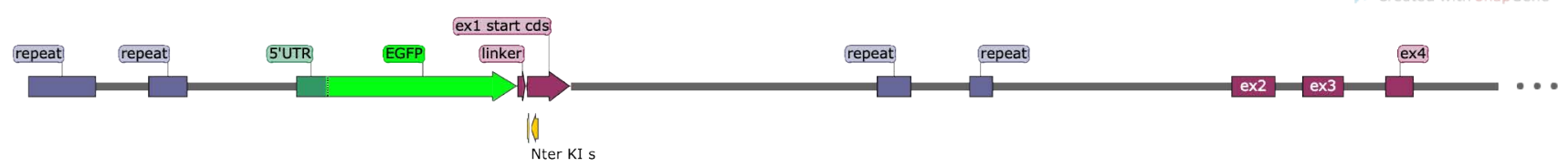

GFP- GFP-

U2OS ATG2A ATG2A

C

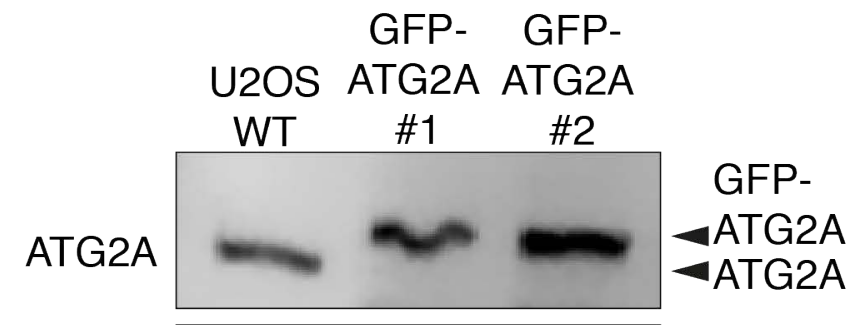

ATG2B

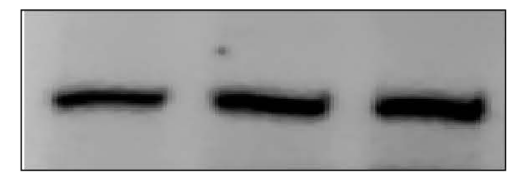

D
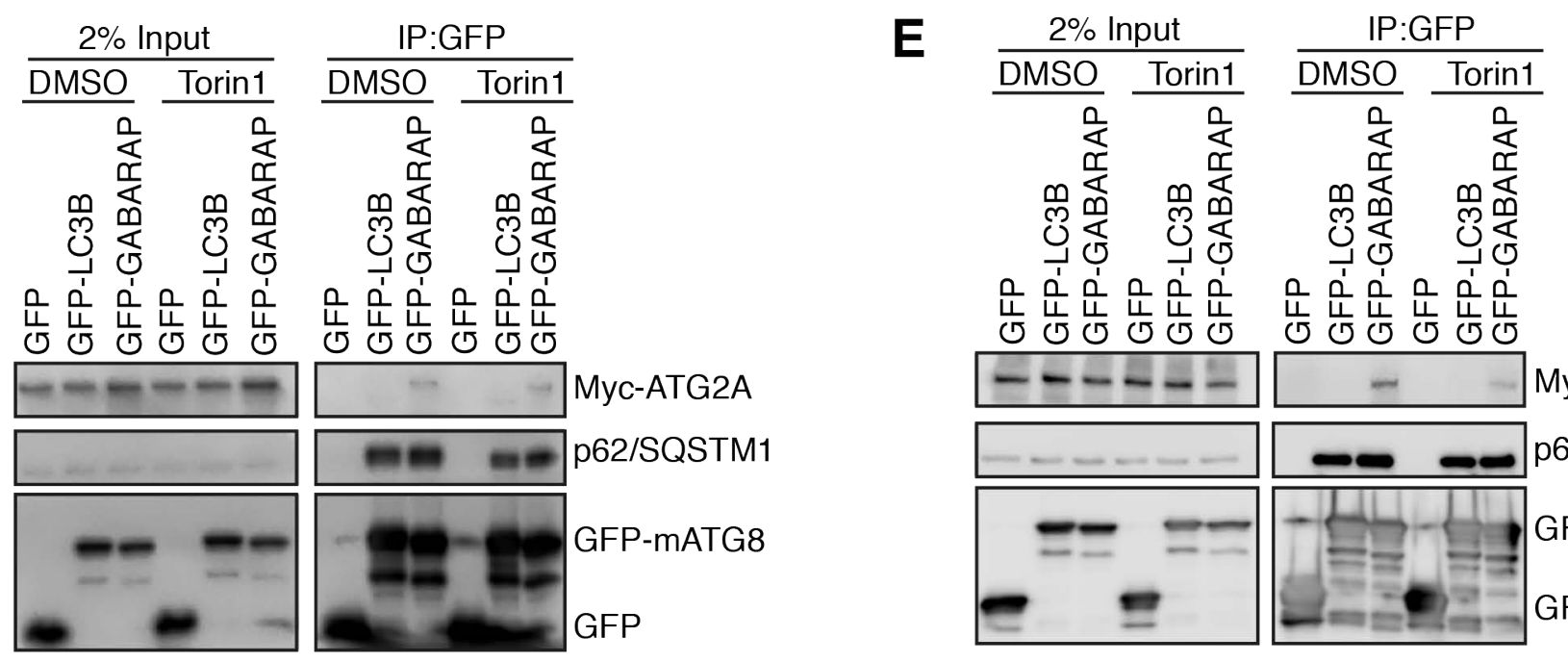

o

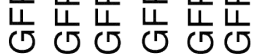

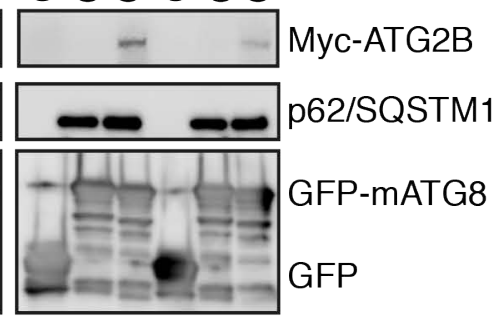

Nter KI s

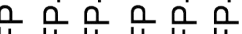

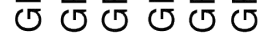

Myc-ATG2B p62/SQSTM1

GFP-mATG8 GFP 

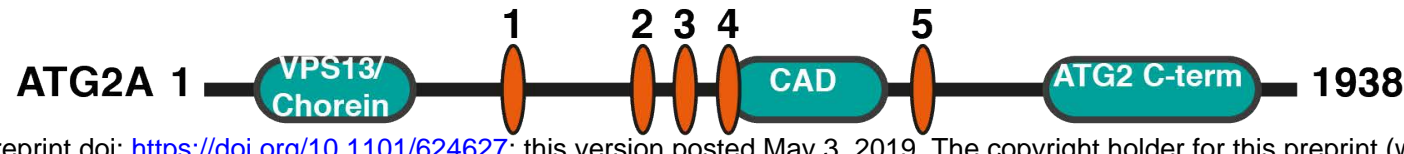

bioRxiv preprint doi: https://doi.org/10.1101/624627; this version posted May 3, 2019. The copyright holder for this preprint (which was not certified by peer review) is the author/funder, who has granted big
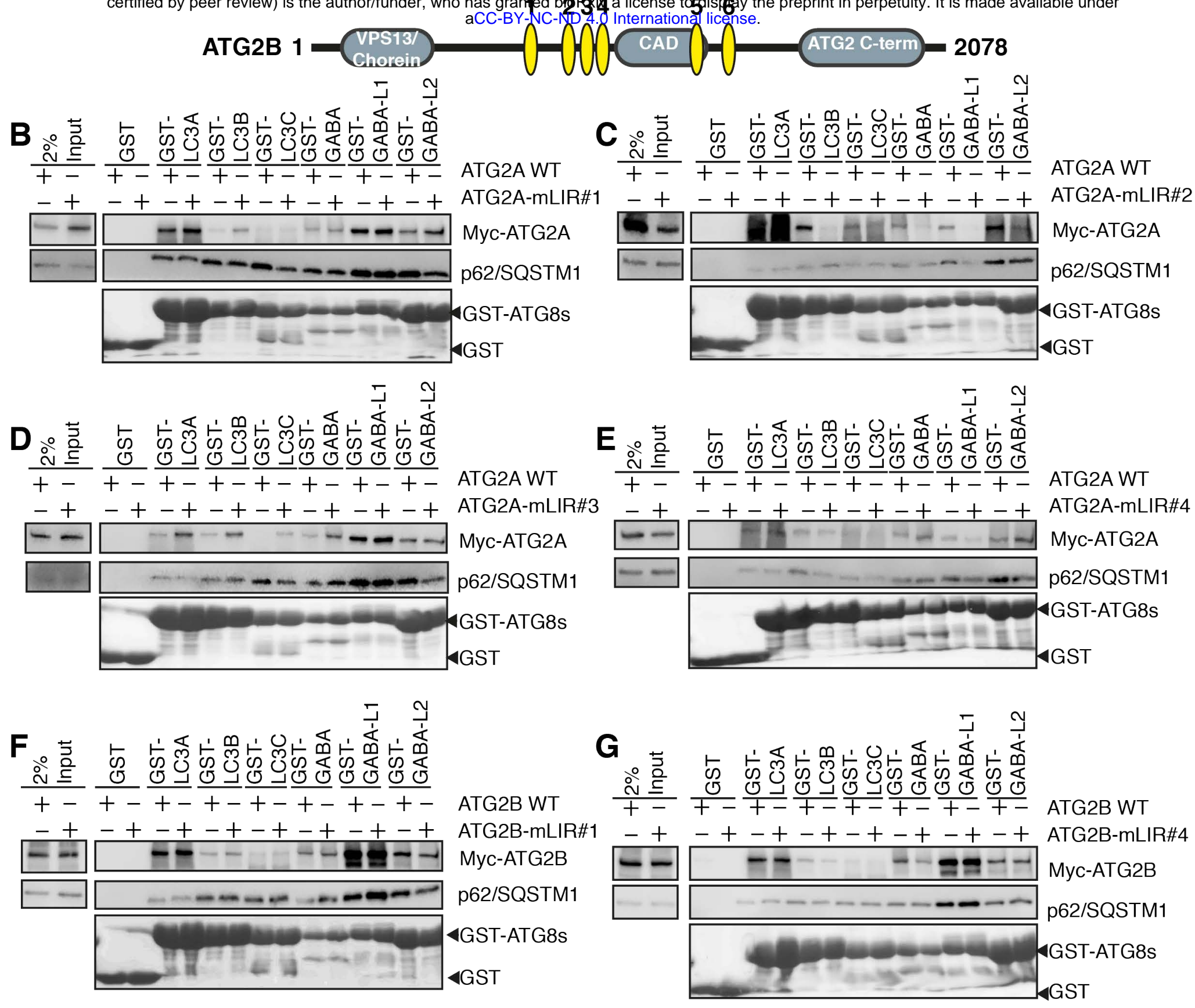

GFP- GFP- GFP-

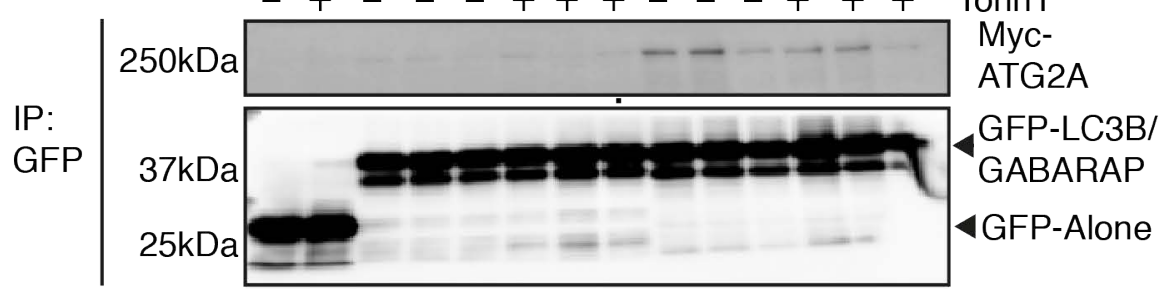

Torin1

ATG2A

GFP-LC3B

GABARAP

4 GFP-Alone

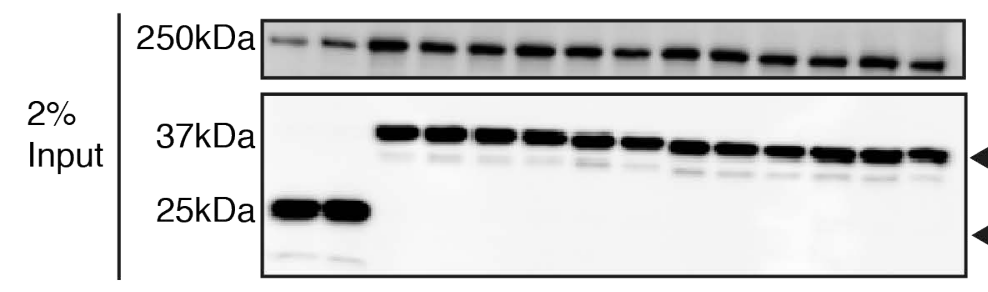

Myc-

ATG2A

GFP-LC3B/

GABARAP

4 GFP-Alone

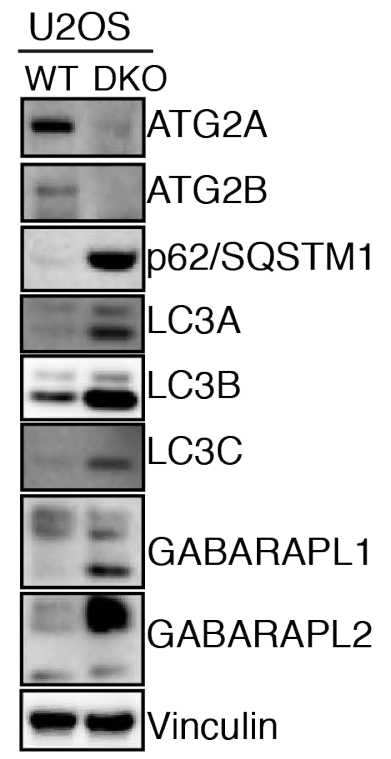


A LC3B/LAMP2/DAPI

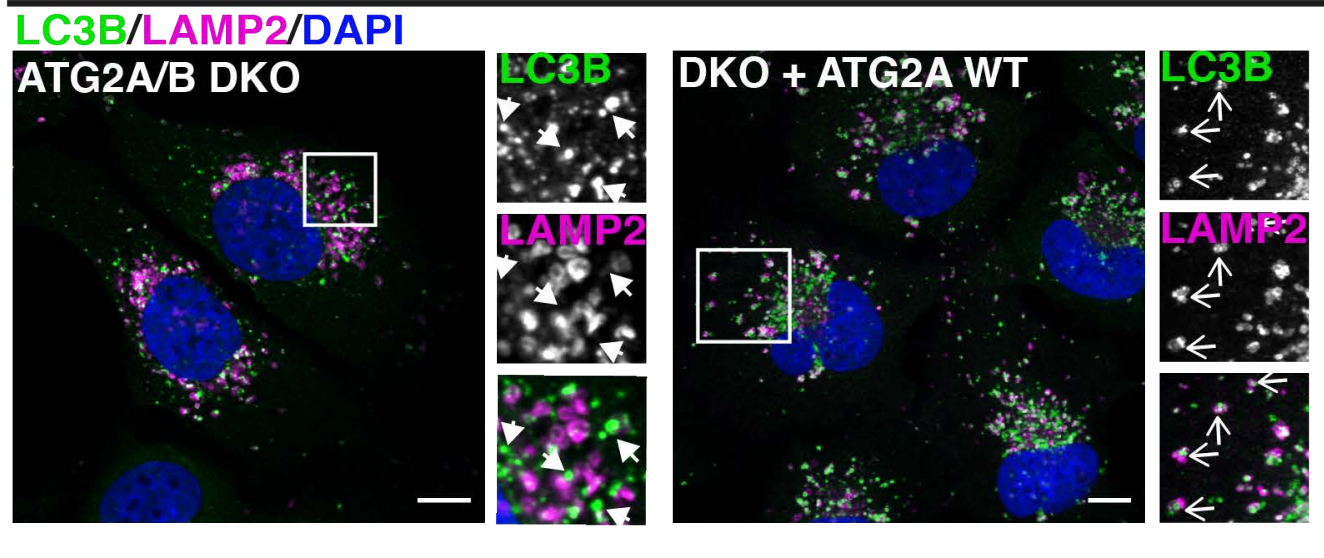

Starve + BafA1
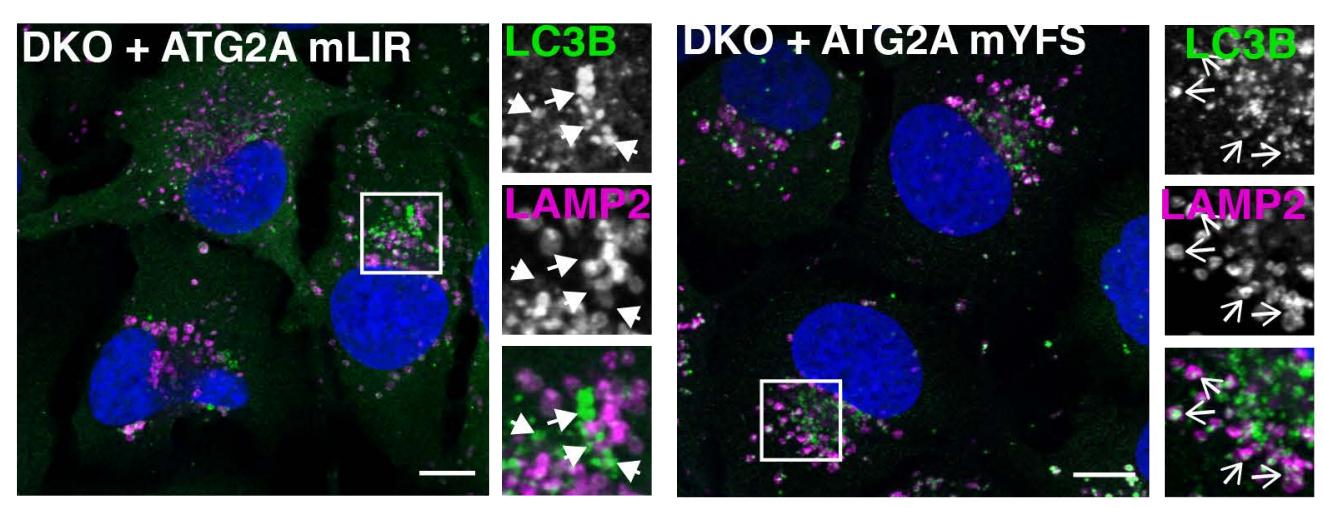

CM+BSA

B Lipid droplets (LD) /HA-ATG2A
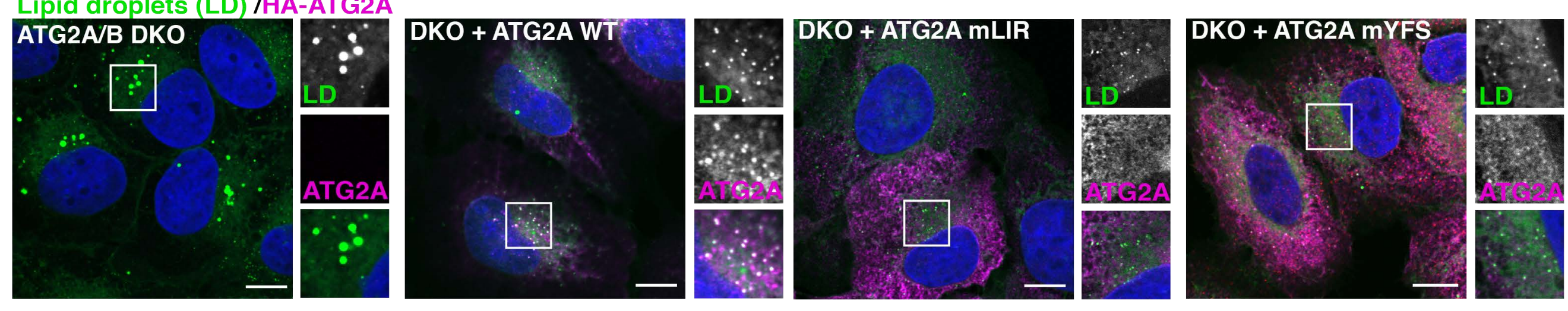

CM + Oleic Acid/BSA

C Lipid droplets (LD) /HA-ATG2A
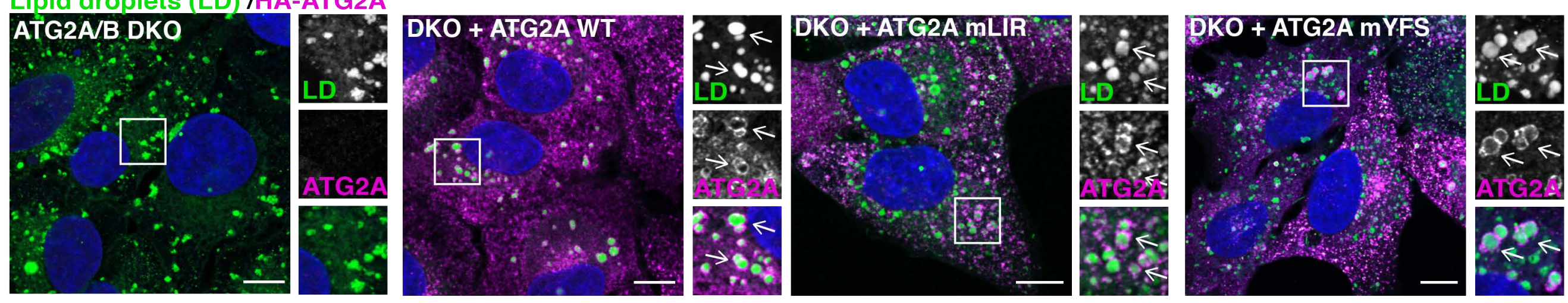
Figure EV4

A LC3B/p62/HA-ATG2A/DAPI

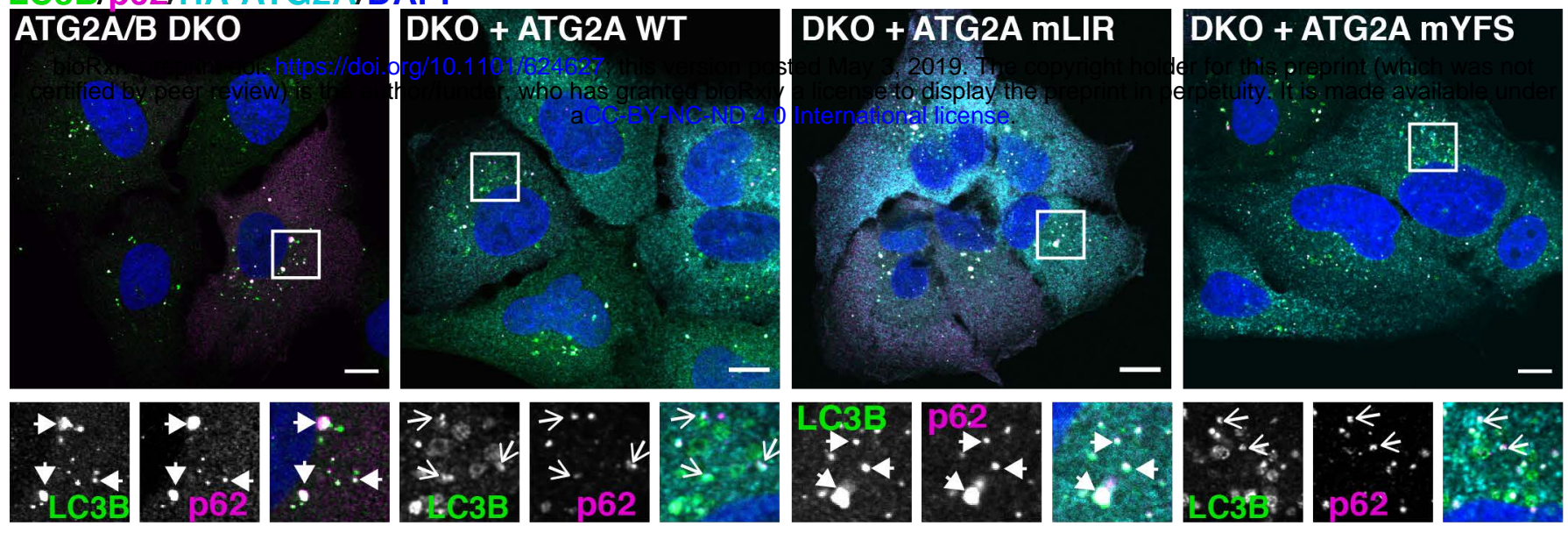

B LC3B/ATG9A/HA-ATG2A/DAPI



C LC3B/WIPI2/HA-ATG2A/DAPI

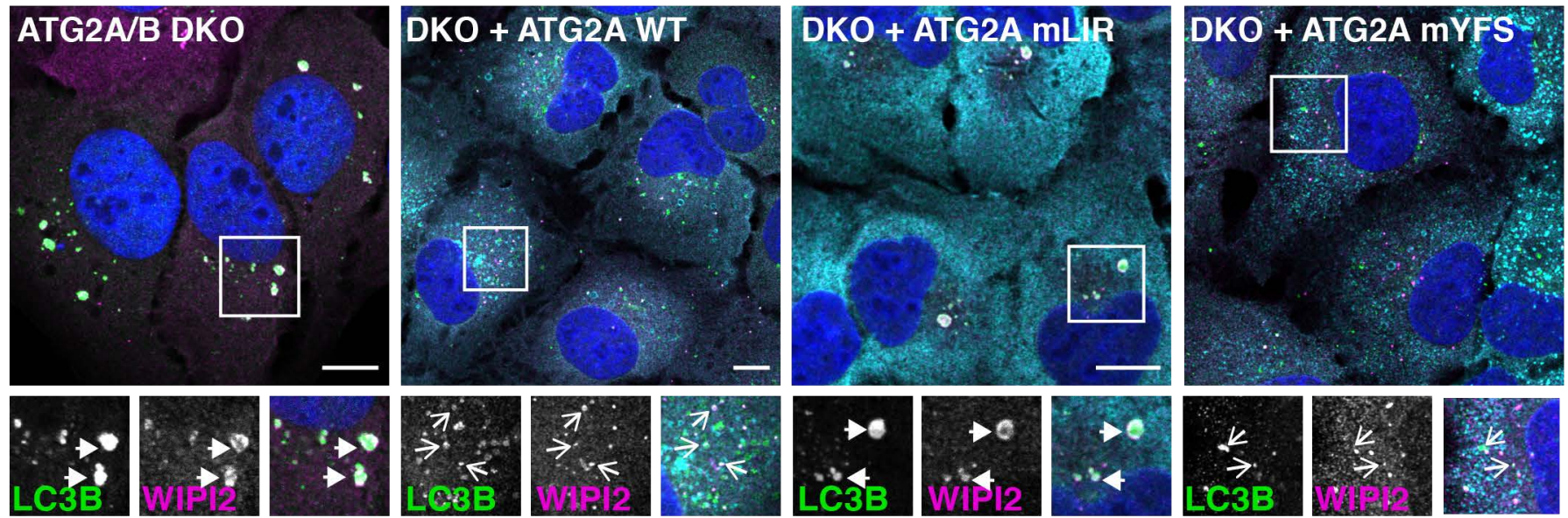

D LC3B/ATG16L1/HA-ATG2A/DAPI

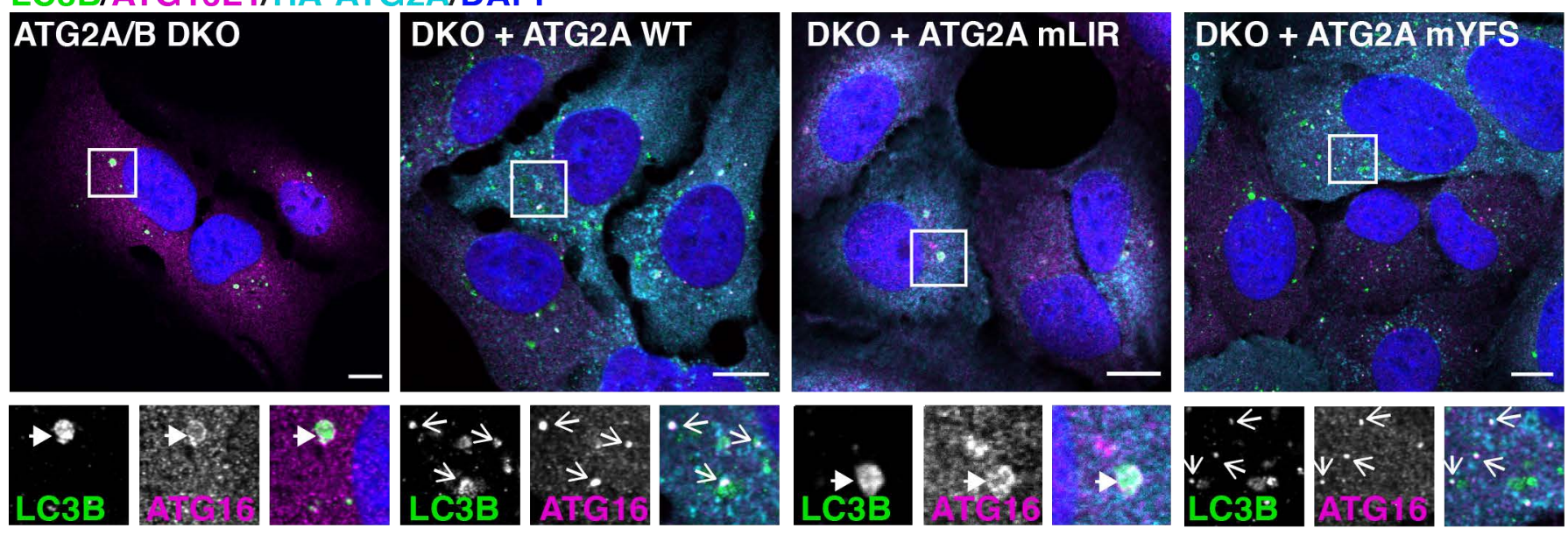

